University of Nebraska - Lincoln

DigitalCommons@University of Nebraska - Lincoln

Papers in the Earth and Atmospheric Sciences

Earth and Atmospheric Sciences, Department

1998

\title{
Dissociation Constants of Oxalic Acid in Aqueous Sodium Chloride and Sodium Trifluoromethanesulfonate Media to $175^{\circ} \mathrm{C}$
}

\author{
Richard Kettler \\ University of Nebraska-Lincoln, rkettler1@unl.edu \\ David J. Wesolowski \\ Oak Ridge National Laboratory \\ Donald A. Palmer \\ Oak Ridge National Laboratory
}

Follow this and additional works at: https://digitalcommons.unl.edu/geosciencefacpub

Part of the Earth Sciences Commons

Kettler, Richard; Wesolowski, David J.; and Palmer, Donald A., "Dissociation Constants of Oxalic Acid in Aqueous Sodium Chloride and Sodium Trifluoromethanesulfonate Media to $175^{\circ} \mathrm{C} "$ (1998). Papers in the Earth and Atmospheric Sciences. 135.

https://digitalcommons.unl.edu/geosciencefacpub/135

This Article is brought to you for free and open access by the Earth and Atmospheric Sciences, Department of at DigitalCommons@University of Nebraska - Lincoln. It has been accepted for inclusion in Papers in the Earth and Atmospheric Sciences by an authorized administrator of DigitalCommons@University of Nebraska - Lincoln. 


\title{
Dissociation Constants of Oxalic Acid in Aqueous Sodium Chloride and Sodium Trifluoromethanesulfonate Media to $175^{\circ} \mathrm{C}$
}

\author{
Richard M. Kettler*,t
}

Department of Geology, University of Nebraska, Lincoln, Nebraska 68588-0340

\author{
David J . Wesolowski and Donald A. Palmer
}

Chemical and Analytical Sciences Division, Oak Ridge National Laboratory, Oak Ridge, Tennessee 37831-6110

\begin{abstract}
The first and second molal dissociation constants of oxalic acid were measured potentiometrically in a concentration cell fitted with hydrogen electrodes. Measurements were made at six temperatures ranging from $5{ }^{\circ} \mathrm{C}$ to $125^{\circ} \mathrm{C}$ at four ionic strengths ranging from $0.1 \mathrm{~mol} \cdot \mathrm{kg}^{-1}$ to $1.0 \mathrm{~mol} \cdot \mathrm{kg}^{-1}\left(\mathrm{NaCl}\right.$ and $\mathrm{NaCF}_{3^{-}}$ $\mathrm{SO}_{3}$ ). The second molal dissociation constant was measured in $\mathrm{NaCF}_{3} \mathrm{SO}_{3}$ media only. The data obtained in this study were combined with the corresponding literature values, including a number of recal culated Harned cell and concentration cell measurements and previous results from this laboratory. Data for first molal dissociation constant obtained in the two ionic media were regressed simultaneously to yield a five-term equation that describes the dissociation in the anionic form. The thermodynamic properties obtained at $25^{\circ} \mathrm{C}$ and infinite dilution are: $\log \mathrm{K}_{1 \mathrm{a}}^{\ominus}=-1.401 \pm 0.052, \Delta \mathrm{H}_{1 \mathrm{a}}=(-0.7 \pm 0.7) \mathrm{kJ} \cdot \mathrm{mol}^{-1}$, $\Delta \mathrm{S}_{1 \mathrm{a}}=(-29 \pm 2) \mathrm{J} \cdot \mathrm{K}^{-1} \cdot \mathrm{mol}^{-1}$, and $\Delta \mathrm{C}_{\mathrm{p}, 1 \mathrm{a}}=(-231 \pm 6) \mathrm{J} \cdot \mathrm{K}^{-1} \cdot \mathrm{mol}^{-1}$. Similar treatment of the original and recalculated data for the second molal dissociation constant yielded a nine-term equation and the following values at $25^{\circ} \mathrm{C}$ and infinite dilution: $\log \mathrm{K}_{2 \mathrm{a}}^{\ominus}=-4.264 \pm 0.014, \Delta \mathrm{H}_{2 \mathrm{a}}=(-7.3 \pm 0.5) \mathrm{kJ} \cdot \mathrm{mol}^{-1}$, $\Delta \mathrm{S}_{2 \mathrm{a}}=(-106 \pm 2) \mathrm{J} \cdot \mathrm{K}^{-1} \cdot \mathrm{mol}^{-1}$, and $\Delta \mathrm{C}_{\mathrm{p}, 2 \mathrm{a}}=(-229 \pm 27) \mathrm{J} \cdot \mathrm{K}^{-1} \cdot \mathrm{mol}^{-1}$.
\end{abstract}

\section{Introduction}

Ethanedioic acid (oxalic acid) is an important biochemical and industrial compound. It is ubiquitous in soils where its concentration is buffered by the presence of the minerals whewellite $\left(\mathrm{CaC}_{2} \mathrm{O}_{4} \cdot \mathrm{H}_{2} \mathrm{O}\right)$ and weddelite $\left(\mathrm{CaC}_{2} \mathrm{O}_{4} \cdot 2 \mathrm{H}_{2} \mathrm{O}\right)$, and it plays a significant role in nutrient availability (Graustein et al., 1977). Because of its ability to form strong complexes with metals, oxalic acid is used to decontaminate the cooling circuits of water-cooled nuclear reactors (Ayres, 1970), and there is considerable interest in the use of oxalic acid to dean kaolin and quartz sand for use by the ceramic industry (Vegliò et al., 1996; Ubaldini et al., 1996). The biochemical, geochemical, and industrial significance of oxalic acid have led to significant interest in the behavior of the compound in high-temperature water (e.g., Bayri et al. (1996)). A recent report (Kettler et al., 1991) from this laboratory examined the dissociation of oxalic acid following the reactions

$$
\mathrm{H}_{2} \mathrm{C}_{2} \mathrm{O}_{4}=\mathrm{HC}_{2} \mathrm{O}_{4}^{-}+\mathrm{H}^{+}
$$

and

$$
\mathrm{HC}_{2} \mathrm{O}_{4}^{-}=\mathrm{C}_{2} \mathrm{O}_{4}^{2-}+\mathrm{H}^{+}
$$

in aqueous sodium chloride media to $175^{\circ} \mathrm{C}$. The respective molal dissociation constants for eqs 1 and 2 are defined as

†Tel: 402-472-2663. Fax: 402-472-4917. E-mail: rkettler@ unlinfo.unl.edu.

₹Tel: 423-574-6903. Fax: 423-574-4971. E-mail: dqw@ornl.gov.

§ Tel: 423-576-5109. Fax: 423-574-4971. E-mail: ddp@ornl.gov.

$$
\mathrm{K}_{\mathrm{m}, \mathrm{aa}}=\left[\mathrm{HC}_{2} \mathrm{O}_{4}^{-}\right]\left[\mathrm{H}^{+}\right] /\left[\mathrm{H}_{2} \mathrm{C}_{2} \mathrm{O}_{4}\right]
$$

and

$$
\mathrm{K}_{\mathrm{m}, 2 \mathrm{a}}=\left[\mathrm{C}_{2} \mathrm{O}_{4}^{2-}\right]\left[\mathrm{H}^{+}\right] /\left[\mathrm{HC}_{2} \mathrm{O}_{4}^{-}\right]
$$

The standard equilibrium constants for eqs 1 and 2 are $\mathrm{K}_{1 \mathrm{a}}^{\ominus}$ and $\mathrm{K}_{2 \mathrm{a}}^{\ominus}$, respectively, and are defined in the usual way (Mills et al., 1993). The standard state used in this paper is a $1 \mathrm{~mol} \cdot \mathrm{kg}^{-1}$ solution behaving as though it were infinitely dilute at the saturation vapor pressure for water at the temperature of interest.

It is difficult to prepare oxalic acid solutions that have low ionic strength: the acid is a strong one and contributes hydrogen, bioxalate, and oxalate ions to the solution. Therefore, the fitting process in the earlier report (Kettler et al., 1991) relied heavily on previously published data (Table 1) in order to extrapolate the molal dissociation constants to infinite dilution. These data included conductance measurements at $25{ }^{\circ} \mathrm{C}$ (Darken, 1941), glass electrode measurements (Kurz and Farrar, 1969), potentiometric data obtained using electrochemical cells without liquid junctions (Parton and Gibbons, 1939; McAuley and Nancollas, 1961), and titration calorimetry measurements (Christensen et al. 1967). The generally accepted value for $\mathrm{pK}_{1 \mathrm{a}, 298}^{\ominus}$ is 1.25 (Martell and Smith, 1977) and is derived largely from the McAuley and Nancollas (1961) measurements (Table 1). The acceptance of a single value for $\mathrm{pK}_{1 \mathrm{a}, 298}^{\ominus}$ belies the wide variety of results reported for the first dissociation at $25^{\circ} \mathrm{C}$ and low values of ionic strength. Measurements in $0.1 \mathrm{~mol} \cdot \mathrm{kg}^{-1} \mathrm{NaClO}_{4}$ yield $\mathrm{pK}_{\mathrm{m}, 1 \mathrm{a}}$ values as high as 1.37 (Table 1) (McAuley and Nancollas, 1960), a value that will increase if extrapolated to infinite dilution. 
338 J ournal of Chemical and Enginering Data, Vol. 43, No. 3, 1998

Table 1. Selected Thermodynamic Data for the Dissociation of Oxalic Acid

\begin{tabular}{|c|c|c|c|c|c|c|}
\hline$-\log K_{m, 1 a}$ & $\Delta \mathrm{H}_{1 \mathrm{a}} /\left(\mathrm{kJ} \cdot \mathrm{mol}^{-1}\right)$ & $-\log K_{m, 2 a}$ & $\Delta \mathrm{H}_{2 \mathrm{a}} /\left(\mathrm{kJ} \cdot \mathrm{mol}^{-1}\right)$ & $\mathrm{t} /{ }^{\circ} \mathrm{C}$ & $\mathrm{I} /\left(\mathrm{mol} \cdot \mathrm{kg}^{-1}\right)$ & reference \\
\hline $1.299^{a}$ & & 4.300 & & 25 & 0 & Parton and Gibbons (1939) \\
\hline $1.271^{\mathrm{a}}$ & & $4.275^{a}$ & & 25 & 0 & Darken (1941) \\
\hline 1.42 & & 4.28 & & 25 & 0 & Choppin and Chen (1996) \\
\hline 1.28 & & 3.65 & & 25 & $0.529\left(\mathrm{NaClO}_{4}\right)$ & Choppin and Chen (1996) \\
\hline 1.33 & & 3.60 & & 25 & $1.073\left(\mathrm{NaClO}_{4}\right)$ & Choppin and Chen (1996) \\
\hline $1.244^{\mathrm{a}}$ & & & & 0 & 0 & McAuley and Nancollas (1961) \\
\hline $1.252^{\mathrm{a}}$ & & & & 15 & 0 & McAuley and Nancollas (1961) \\
\hline $1.252^{\mathrm{a}}$ & & & & 25 & 0 & McAuley and Nancollas (1961) \\
\hline $1.286^{a}$ & & & & 35 & 0 & McAuley and Nancollas (1961) \\
\hline $1.295^{\mathrm{a}}$ & & & & 45 & 0 & McAuley and Nancollas (1961) \\
\hline $1.30^{\mathrm{a}}$ & -3.35 & & & 25 & 0 & Kurz and Farrar (1969) \\
\hline $1.31^{a}$ & & & & 30 & 0 & Kurz and Farrar (1969) \\
\hline $1.32^{\mathrm{a}}$ & & & & 35 & 0 & Kurz and Farrar (1969) \\
\hline $1.33^{\mathrm{a}}$ & & & & 40 & 0 & Kurz and Farrar (1969) \\
\hline $1.34^{\mathrm{a}}$ & & & & 45 & 0 & Kurz and Farrar (1969) \\
\hline $1.36^{a}$ & & & & 50 & 0 & Kurz and Farrar (1969) \\
\hline $1.36^{a}$ & & & & 55 & 0 & Kurz and Farrar (1969) \\
\hline & & $4.228^{a}$ & -1.16 & 0 & 0 & Harned and Fallon (1939) \\
\hline & & $4.235^{a}$ & -2.10 & 5 & 0 & Harned and Fallon (1939) \\
\hline & & $4.244^{a}$ & -4.15 & 10 & 0 & Harned and Fallon (1939) \\
\hline & & $4.255^{a}$ & -5.29 & 15 & 0 & Harned and Fallon (1939) \\
\hline & & $4.268^{a}$ & -5.29 & 20 & 0 & Harned and Fallon (1939) \\
\hline & & $4.286^{a}$ & -6.49 & 25 & 0 & Harned and Fallon (1939) \\
\hline & & $4.308^{a}$ & -7.77 & 30 & 0 & Harned and Fallon (1939) \\
\hline & & $4.331^{a}$ & -9.11 & 35 & 0 & Harned and Fallon (1939) \\
\hline & & $4.356^{a}$ & -10.54 & 40 & 0 & Harned and Fallon (1939) \\
\hline & & $4.388^{a}$ & -12.04 & 45 & 0 & Harned and Fallon (1939) \\
\hline & & $4.417^{a}$ & -13.62 & 50 & 0 & Harned and Fallon (1939) \\
\hline & & $4.201^{a}$ & -1.447 & 0 & 0 & Pinching and Bates (1948) \\
\hline & & $4.207^{a}$ & -2.508 & 5 & 0 & Pinching and Bates (1948) \\
\hline & & $4.218^{a}$ & -3.587 & 10 & 0 & Pinching and Bates (1948) \\
\hline & & $4.231^{a}$ & -4.686 & 15 & 0 & Pinching and Bates (1948) \\
\hline & & $4.247^{a}$ & -5.804 & 20 & 0 & Pinching and Bates (1948) \\
\hline & & $4.266^{a}$ & -6.941 & 25 & 0 & Pinching and Bates (1948) \\
\hline & & $4.287^{a}$ & -8.098 & 30 & 0 & Pinching and Bates (1948) \\
\hline & & $4.312^{\mathrm{a}}$ & -9.274 & 35 & 0 & Pinching and Bates (1948) \\
\hline & & $4.338^{a}$ & -10.47 & 40 & 0 & Pinching and Bates (1948) \\
\hline & & $4.369^{a}$ & -11.68 & 45 & 0 & Pinching and Bates (1948) \\
\hline & & 4.399a & -12.92 & 50 & 0 & Pinching and Bates (1948) \\
\hline & $-4.27 \pm 0.21^{a}$ & & $-6.28 \pm 0.21^{a}$ & 25 & 0 & Christensen et al. (1967) \\
\hline 1.37 & & 3.81 & & 25 & $0.10\left(\mathrm{NaClO}_{4}\right)$ & McAuley and Nancollas (1960) \\
\hline 1.33 & & 3.94 & & 25 & $0.15\left(\mathrm{NaClO}_{4}\right)$ & de Bruin et al. (1962) \\
\hline 1.20 & & 3.67 & & 25 & $0.50\left(\mathrm{NaClO}_{4}\right)$ & Bauer and Smith (1965) \\
\hline 1.07 & & 3.57 & & 25 & $1.0\left(\mathrm{NaClO}_{4}\right)$ & Bottari and Ciavatta (1965) \\
\hline 1.08 & & 3.554 & & 25 & $1.0\left(\mathrm{NaClO}_{4}\right)$ & Moorhead and Sutin (1966) \\
\hline 1.1 & & 3.62 & & 25 & $1.0\left(\mathrm{KNO}_{3}\right)$ & Rajan and Martell (1967) \\
\hline 0.89 & & 3.51 & & 25 & $1.0(\mathrm{NaCl})$ & Cruywagen et al. (1986) \\
\hline
\end{tabular}

a Datum was used in the fitting process by Kettler et al. (1991).

Moreover, Choppin and Chen (1996) have reported a value of 1.42 for $\mathrm{pK}_{1 \mathrm{a}, 298}^{\ominus}$ recently.

Subsequent to the earlier report (Kettler et al., 1991), measurements of $K_{m, 1 a}$ and $K_{m, 2 a}$ were made in aqueous sodium trifluoromethanesulfonate ( $\mathrm{Na}$-triflate) media in this laboratory. These later measurements are inconsistent with the low-temperature $\left(<75{ }^{\circ} \mathrm{C}\right)$ extrapolations published in the earlier report and have prompted a reexamination of the data in the earlier report as well as those data available in the literature for this system. This paper reports the measurement of $\mathrm{K}_{\mathrm{m}, 1 \mathrm{a}}$ and $\mathrm{K}_{\mathrm{m}, 2 \mathrm{a}}$ in $\mathrm{Na}$ triflate media, the results of additional measurements of $\mathrm{K}_{\mathrm{m}, 1 \mathrm{a}}$ in aqueous $\mathrm{NaCl}$ media, and the results of a fitting process that incorporates values obtained by recalculating and refitting previously published data. An improved set of equations describing the dissociation of oxalic acid is given as is a significant revision in the widely accepted value for $\mathrm{pK}_{1 \mathrm{a}}^{\ominus}$ at $25^{\circ} \mathrm{C}$.

\section{Experimental Section}

Materials. Stock solutions of $\mathrm{HCl}, \mathrm{NaOH}$, and $\mathrm{NaCl}$ were prepared using reagent-grade chemicals and water that had been purified in a Barnstead four-stage deionizing/ purification system to yield water with a resistivity of 0.18 M $\Omega \cdot m$ (Palmer and Wesolowski, 1993). The stock solutions were sparged and stored under argon. Concentrated reagent-grade (Aldrich Chemical Co., lot AQ06628DN) trifluoromethanesulfonic (triflic) acid was purified by vacuum distillation (Palmer and Drummond, 1988). Crystalline $\mathrm{Na}$-triflate was synthesized by reacting concentrated triflic acid with an equivalent amount of concentrated $\mathrm{NaOH}$ and recrystall izing the resulting solid from an ethanolic solution (Palmer and Drummond, 1988). Stock solutions of the acid and salt were prepared by combining the purified analyte with water. Stock solutions of oxalic acid were prepared from reagent-grade $\mathrm{H}_{2} \mathrm{C}_{2} \mathrm{O}_{4} \cdot 2 \mathrm{H}_{2} \mathrm{O}$ (Mallinckrodt, lot NTP) and water. The concentrations of all stock solutions were measured by acidimetric titration (after cation exchange when necessary) with the $\mathrm{NaOH}$ stock solution.

Ratios of total oxalate to supporting el ectrolyte ranged from 1:8.6 in the most dilute solutions to 1:94 in the more concentrated solutions (Tables 2 and 3 ). Ratios of acid to supporting electrolyte in the reference solutions were varied depending on whether the experiments were designed to measure the first or second molal dissociation 
Table 2. Starting Molal Solution Compositions for Experiments in Aqueous Sodium Chloride Media

\begin{tabular}{lcccccc}
\hline & \multicolumn{3}{c}{ test cell } & & \multicolumn{2}{c}{ reference cell } \\
\cline { 2 - 3 } & $\mathrm{m}(\mathrm{NaCl}) /\left(\mathrm{mol} \cdot \mathrm{kg}^{-1}\right)$ & $\mathrm{m}\left(\mathrm{H}_{2} \mathrm{C}_{2} \mathrm{O}_{4}\right) /\left(\mathrm{mol} \cdot \mathrm{kg}^{-1}\right)$ & $\mathrm{m}(\mathrm{HCl}) /\left(\mathrm{mol} \cdot \mathrm{kg}^{-1}\right)$ & & $\mathrm{m}(\mathrm{HCl}) /\left(\mathrm{mol} \cdot \mathrm{kg}^{-1}\right)$ & $\mathrm{m}(\mathrm{NaCl}) /\left(\mathrm{mol} \cdot \mathrm{kg}^{-1}\right)$ \\
\hline 20,40 & 0.086483 & 0.01001 & 0.004999 & 0.005143 & 0.094864 \\
37,38 & 0.30176 & 0.0099935 & 0.0051038 & & 0.0049998 & 0.31002 \\
49 & 0.94241 & 0.014993 & 0.049957 & 0.050019 & 0.95069 \\
51 & 0.56499 & 0.008989 & 0.029950 & & 0.029992 & 0.57004 \\
52 & 0.56455 & 0.008982 & 0.029927 & & 0.029980 & 0.56982
\end{tabular}

Table 3. Starting Molal Solution Compositions for Experiments in Aqueous Sodium Trifluoromethanesulfonate Media

\begin{tabular}{|c|c|c|c|c|c|c|}
\hline \multirow[b]{2}{*}{ expt } & \multicolumn{4}{|c|}{ test cell } & \multicolumn{2}{|c|}{ reference cell } \\
\hline & $\mathrm{m}(\mathrm{NaTr}) /\left(\mathrm{mol} \cdot \mathrm{kg}^{-1}\right)$ & $\mathrm{m}\left(\mathrm{H}_{2} \mathrm{C}_{2} \mathrm{O}_{4}\right) /\left(\mathrm{mol} \cdot \mathrm{kg}^{-1}\right)$ & $\mathrm{m}(\mathrm{HTr}) /\left(\mathrm{mol} \cdot \mathrm{kg}^{-1}\right)$ & $\mathrm{m}(\mathrm{NaOH}) /\left(\mathrm{mol} \cdot \mathrm{kg}^{-1}\right)$ & $\mathrm{m}(\mathrm{HTr}) /\left(\mathrm{mol} \cdot \mathrm{kg}^{-1}\right)$ & $\mathrm{m}(\mathrm{NaTr}) /\left(\mathrm{mol} \cdot \mathrm{kg}^{-1}\right)$ \\
\hline 6 & 0.07957 & 0.01024 & & 0.01527 & 0.005018 & 0.09506 \\
\hline 8 & 0.07938 & 0.01025 & & 0.01537 & 0.005018 & 0.09507 \\
\hline 16,17 & 0.08790 & 0.01020 & 0.005097 & & 0.005018 & 0.09507 \\
\hline 18,19 & 0.08790 & 0.01020 & 0.005097 & & 0.004999 & 0.09505 \\
\hline 21,22 & 0.3019 & 0.01000 & 0.005004 & & 0.005263 & 0.3106 \\
\hline 23,24 & 0.5858 & 0.01001 & 0.005004 & & 0.004999 & 0.5949 \\
\hline 25,26 & 0.9444 & 0.009999 & 0.04999 & & 0.05000 & 0.9500 \\
\hline 27 & 0.07998 & 0.01001 & & 0.01502 & 0.09999 & 0.09833 \\
\hline 28 & 0.07998 & 0.01001 & & 0.01502 & 0.0008331 & 0.09914 \\
\hline 44 & 0.9698 & 0.01499 & & 0.02209 & 0.004898 & 0.9963 \\
\hline 45 & 0.5822 & 0.008998 & & 0.01326 & 0.002934 & 0.5968 \\
\hline 46 & 0.2530 & 0.009995 & & 0.01479 & 0.001335 & 0.2716 \\
\hline 47 & 0.5824 & 0.009002 & & 0.01326 & 0.004999 & 0.5949 \\
\hline 48 & 0.3065 & 0.004737 & & 0.006980 & 0.005263 & 0.3106 \\
\hline
\end{tabular}

Table 4. Experimental Results for the First Dissociation of Oxalic Acid in Aqueous Sodium Chloride Media

\begin{tabular}{|c|c|c|c|c|c|c|c|c|c|c|c|}
\hline expt & $\mathrm{t} /{ }^{\circ} \mathrm{C}$ & $\mathrm{I} /\left(\mathrm{mol} \cdot \mathrm{kg}^{-1}\right)$ & $\mathrm{E} \cdot 10^{3} \mathrm{~N}$ & $\mathrm{E}_{\mathrm{lj}}{ }^{\mathrm{a}} \cdot 10^{3} \mathrm{~N}$ & $\mathrm{pH}^{\mathrm{b}}$ & $\bar{n}$ & $\sigma_{\text {กิ }}$ & $\log K_{m, 1 a}$ & $\sigma_{1 a}$ & $\log K_{m, 1 b}$ & $\sigma_{1 \mathrm{~b}}$ \\
\hline \multirow[t]{6}{*}{20} & 4.96 & 0.100 & -17.83 & -4.68 & 1.881 & 1.185 & 0.108 & -1.271 & 0.296 & 13.249 & 0.302 \\
\hline & 24.89 & 0.100 & -19.64 & -4.40 & 1.882 & 1.190 & 0.089 & -1.282 & 0.235 & 12.497 & 0.241 \\
\hline & 49.77 & 0.100 & -21.73 & -4.07 & 1.886 & 1.201 & 0.072 & -1.310 & 0.183 & 11.738 & 0.189 \\
\hline & 74.81 & 0.099 & -23.69 & -3.73 & 1.891 & 1.217 & 0.059 & -1.351 & 0.142 & 11.117 & 0.148 \\
\hline & 100.10 & 0.099 & -25.65 & -3.41 & 1.896 & 1.231 & 0.048 & -1.385 & 0.112 & 10.613 & 0.118 \\
\hline & 125.12 & 0.099 & -26.78 & -3.10 & 1.910 & 1.272 & 0.039 & -1.489 & 0.083 & 10.136 & 0.089 \\
\hline \multirow[t]{6}{*}{37} & 5.03 & 0.316 & -22.66 & -1.88 & 1.856 & 1.118 & 0.035 & -1.051 & 0.098 & 13.402 & 0.107 \\
\hline & 24.84 & 0.316 & -24.46 & -1.73 & 1.858 & 1.123 & 0.029 & -1.068 & 0.080 & 12.645 & 0.089 \\
\hline & 49.85 & 0.316 & -26.65 & -1.57 & 1.861 & 1.132 & 0.025 & -1.089 & 0.066 & 11.882 & 0.075 \\
\hline & 74.90 & 0.316 & -28.52 & -1.41 & 1.868 & 1.154 & 0.020 & -1.159 & 0.051 & 11.222 & 0.060 \\
\hline & 100.03 & 0.315 & -30.21 & -1.25 & 1.876 & 1.180 & 0.017 & -1.238 & 0.039 & 10.665 & 0.048 \\
\hline & 124.98 & 0.315 & -31.51 & -1.13 & 1.887 & 1.215 & 0.014 & -1.338 & 0.031 & 10.178 & 0.040 \\
\hline \multirow[t]{6}{*}{38} & 5.12 & 0.316 & -22.67 & -1.88 & 1.856 & 1.118 & 0.035 & -1.050 & 0.098 & 13.399 & 0.107 \\
\hline & 24.96 & 0.316 & -24.49 & -1.73 & 1.858 & 1.122 & 0.029 & -1.064 & 0.081 & 12.645 & 0.090 \\
\hline & 49.91 & 0.316 & -26.65 & -1.57 & 1.861 & 1.132 & 0.025 & -1.090 & 0.066 & 11.879 & 0.075 \\
\hline & 75.01 & 0.316 & -28.55 & -1.41 & 1.867 & 1.153 & 0.020 & -1.156 & 0.051 & 11.223 & 0.060 \\
\hline & 100.03 & 0.315 & -30.20 & -1.25 & 1.876 & 1.180 & 0.017 & -1.240 & 0.039 & 10.663 & 0.048 \\
\hline & 125.01 & 0.315 & -31.49 & -1.13 & 1.887 & 1.216 & 0.014 & -1.340 & 0.031 & 10.175 & 0.040 \\
\hline \multirow[t]{6}{*}{40} & 5.06 & 0.100 & -18.20 & -4.81 & 1.872 & 1.158 & 0.114 & -1.183 & 0.369 & 13.329 & 0.375 \\
\hline & 24.94 & 0.100 & -20.10 & -4.53 & 1.872 & 1.159 & 0.095 & -1.184 & 0.294 & 12.590 & 0.300 \\
\hline & 49.84 & 0.100 & -22.25 & -4.19 & 1.876 & 1.171 & 0.077 & -1.216 & 0.222 & 11.826 & 0.228 \\
\hline & 74.82 & 0.100 & -24.13 & -3.82 & 1.884 & 1.194 & 0.061 & -1.284 & 0.162 & 11.180 & 0.168 \\
\hline & 100.08 & 0.099 & -25.78 & -3.43 & 1.894 & 1.225 & 0.049 & -1.369 & 0.116 & 10.626 & 0.122 \\
\hline & 125.02 & 0.099 & -26.94 & -3.12 & 1.908 & 1.265 & 0.040 & -1.472 & 0.086 & 10.150 & 0.092 \\
\hline \multirow[t]{6}{*}{49} & 5.06 & 1.002 & -3.65 & -0.60 & 1.224 & 1.349 & 0.036 & -0.961 & 0.066 & 13.499 & 0.076 \\
\hline & 24.89 & 1.002 & -4.05 & -0.57 & 1.223 & 1.339 & 0.032 & -0.941 & 0.059 & 12.775 & 0.069 \\
\hline & 49.84 & 1.002 & -4.29 & -0.50 & 1.226 & 1.369 & 0.027 & -1.000 & 0.047 & 11.967 & 0.057 \\
\hline & 74.63 & 1.002 & -4.48 & -0.44 & 1.229 & 1.400 & 0.022 & -1.059 & 0.039 & 11.309 & 0.049 \\
\hline & 100.10 & 1.001 & -4.61 & -0.39 & 1.233 & 1.435 & 0.019 & -1.124 & 0.033 & 10.739 & 0.043 \\
\hline & 125.02 & 1.002 & -4.56 & -0.34 & 1.238 & 1.484 & 0.017 & -1.214 & 0.029 & 10.241 & 0.039 \\
\hline \multirow[t]{6}{*}{51} & 5.07 & 0.602 & -4.15 & -0.69 & 1.435 & 1.250 & 0.042 & -0.974 & 0.089 & 13.466 & 0.098 \\
\hline & 24.88 & 0.602 & -4.56 & -0.64 & 1.435 & 1.246 & 0.036 & -0.966 & 0.078 & 12.732 & 0.087 \\
\hline & 49.89 & 0.602 & -4.86 & -0.57 & 1.438 & 1.277 & 0.030 & -1.033 & 0.061 & 11.918 & 0.070 \\
\hline & 74.94 & 0.601 & -5.04 & -0.50 & 1.443 & 1.319 & 0.025 & -1.122 & 0.047 & 11.233 & 0.056 \\
\hline & 100.06 & 0.601 & -5.27 & -0.44 & 1.446 & 1.347 & 0.021 & -1.178 & 0.039 & 10.690 & 0.048 \\
\hline & 125.00 & 0.601 & -5.28 & -0.39 & 1.451 & 1.396 & 0.019 & -1.272 & 0.033 & 10.198 & 0.042 \\
\hline \multirow[t]{6}{*}{52} & 5.04 & 0.601 & -4.11 & -0.68 & 1.436 & 1.256 & 0.041 & -0.989 & 0.086 & 13.453 & 0.095 \\
\hline & 24.89 & 0.601 & -4.58 & -0.65 & 1.435 & 1.241 & 0.037 & -0.953 & 0.079 & 12.745 & 0.088 \\
\hline & 49.91 & 0.601 & -4.85 & -0.57 & 1.439 & 1.276 & 0.030 & -1.033 & 0.061 & 11.918 & 0.070 \\
\hline & 74.91 & 0.601 & -5.03 & -0.50 & 1.443 & 1.318 & 0.025 & -1.121 & 0.047 & 11.234 & 0.056 \\
\hline & 100.07 & 0.601 & -5.26 & -0.44 & 1.446 & 1.347 & 0.021 & -1.177 & 0.039 & 10.690 & 0.048 \\
\hline & 124.91 & 0.601 & -5.26 & -0.39 & 1.451 & 1.397 & 0.019 & -1.273 & 0.033 & 10.199 & 0.042 \\
\hline
\end{tabular}

\footnotetext{
a Liquid junction potential, see text. ${ }^{b}$ Defined as $-\log \left[\mathrm{H}^{+}\right]$.
} 
340 J ournal of Chemical and Engineering Data, Vol. 43, No. 3, 1998

Table 5. Experimental Results for the First Dissociation of Oxalic Acid in Aqueous Sodium Trifluoromethanesulfonate Mediaa

\begin{tabular}{|c|c|c|c|c|c|c|c|c|c|c|c|}
\hline expt & $\mathrm{t} /{ }^{\circ} \mathrm{C}$ & $\mathrm{I} /\left(\mathrm{mol} \cdot \mathrm{kg}^{-1}\right)$ & $\mathrm{E} \cdot 10^{3} \mathrm{~N}$ & $\mathrm{E}_{\mathrm{lj}} \cdot 10^{3} \mathrm{~N}$ & $\mathrm{pH}$ & $\bar{n}$ & $\sigma_{\text {กิ }}$ & $\log K_{m, 1 a}$ & $\sigma_{1 a}$ & $\log K_{m, 1 b}$ & $\sigma_{1 \mathrm{~b}}$ \\
\hline \multirow[t]{5}{*}{16} & 24.87 & 0.101 & -20.02 & -5.36 & 1.870 & 1.178 & 0.052 & -1.236 & 0.150 & 12.557 & 0.156 \\
\hline & 49.87 & 0.101 & -22.46 & -4.93 & 1.872 & 1.184 & 0.043 & -1.248 & 0.122 & 11.811 & 0.128 \\
\hline & 75.03 & 0.101 & -24.66 & -4.33 & 1.880 & 1.207 & 0.034 & -1.311 & 0.088 & 11.166 & 0.094 \\
\hline & 100.09 & 0.101 & -26.49 & -3.70 & 1.892 & 1.242 & 0.026 & -1.405 & 0.060 & 10.607 & 0.066 \\
\hline & 124.88 & 0.101 & -27.84 & -3.27 & 1.905 & 1.281 & 0.021 & -1.503 & 0.045 & 10.138 & 0.051 \\
\hline \multirow[t]{4}{*}{17} & 5.00 & 0.101 & -17.79 & -5.42 & 1.879 & 1.204 & 0.055 & -1.318 & 0.142 & 13.215 & 0.148 \\
\hline & 24.83 & 0.101 & -19.98 & -5.35 & 1.871 & 1.180 & 0.052 & -1.244 & 0.147 & 12.551 & 0.153 \\
\hline & 49.90 & 0.101 & -22.49 & -4.93 & 1.872 & 1.182 & 0.043 & -1.242 & 0.123 & 11.817 & 0.129 \\
\hline & 74.87 & 0.101 & -24.68 & -4.35 & 1.879 & 1.205 & 0.034 & -1.305 & 0.089 & 11.176 & 0.095 \\
\hline \multirow[t]{6}{*}{18} & 4.98 & 0.101 & -17.98 & -5.49 & 1.876 & 1.194 & 0.056 & -1.290 & 0.150 & 13.243 & 0.156 \\
\hline & 24.83 & 0.102 & -20.22 & -5.43 & 1.867 & 1.169 & 0.053 & -1.206 & 0.160 & 12.588 & 0.166 \\
\hline & 49.91 & 0.102 & -22.77 & -5.02 & 1.868 & 1.170 & 0.044 & -1.203 & 0.134 & 11.855 & 0.140 \\
\hline & 74.91 & 0.101 & -24.92 & -4.40 & 1.876 & 1.197 & 0.034 & -1.282 & 0.093 & 11.198 & 0.099 \\
\hline & 100.11 & 0.101 & -26.71 & -3.74 & 1.890 & 1.236 & 0.026 & -1.390 & 0.062 & 10.622 & 0.068 \\
\hline & 125.03 & 0.101 & -28.01 & -3.29 & 1.904 & 1.279 & 0.021 & -1.498 & 0.045 & 10.140 & 0.051 \\
\hline \multirow[t]{5}{*}{19} & 5.02 & 0.101 & -17.99 & -5.50 & 1.876 & 1.194 & 0.056 & -1.289 & 0.151 & 13.243 & 0.157 \\
\hline & 24.89 & 0.101 & -20.12 & -5.38 & 1.870 & 1.176 & 0.052 & -1.231 & 0.152 & 12.561 & 0.158 \\
\hline & 49.99 & 0.101 & -22.61 & -4.96 & 1.871 & 1.181 & 0.043 & -1.238 & 0.124 & 11.818 & 0.130 \\
\hline & 74.87 & 0.101 & -24.69 & -4.34 & 1.881 & 1.209 & 0.034 & -1.319 & 0.087 & 11.161 & 0.093 \\
\hline & 100.09 & 0.101 & -26.63 & -3.72 & 1.891 & 1.240 & 0.026 & -1.400 & 0.061 & 10.611 & 0.067 \\
\hline \multirow[t]{5}{*}{21} & 5.03 & 0.316 & -20.87 & -2.14 & 1.862 & 1.126 & 0.018 & -1.084 & 0.065 & 13.381 & 0.074 \\
\hline & 24.86 & 0.316 & -22.71 & -2.03 & 1.860 & 1.121 & 0.017 & -1.060 & 0.062 & 12.664 & 0.071 \\
\hline & 49.89 & 0.316 & -24.84 & -1.81 & 1.863 & 1.130 & 0.014 & -1.082 & 0.051 & 11.902 & 0.060 \\
\hline & 74.87 & 0.316 & -26.69 & -1.55 & 1.870 & 1.151 & 0.012 & -1.150 & 0.037 & 11.248 & 0.046 \\
\hline & 100.10 & 0.315 & -28.19 & -1.30 & 1.880 & 1.184 & 0.009 & -1.253 & 0.026 & 10.667 & 0.035 \\
\hline \multirow[t]{3}{*}{22} & 4.94 & 0.316 & -20.86 & -2.14 & 1.862 & 1.126 & 0.018 & -1.085 & 0.065 & 13.385 & 0.074 \\
\hline & 24.78 & 0.316 & -22.75 & -2.04 & 1.859 & 1.118 & 0.017 & -1.048 & 0.063 & 12.679 & 0.072 \\
\hline & 49.86 & 0.316 & -24.83 & -1.81 & 1.863 & 1.130 & 0.014 & -1.084 & 0.051 & 11.902 & 0.060 \\
\hline \multirow[t]{4}{*}{23} & 5.02 & 0.600 & -23.09 & -1.23 & 1.861 & 1.123 & 0.011 & -1.082 & 0.039 & 13.374 & 0.048 \\
\hline & 24.87 & 0.600 & -25.04 & -1.16 & 1.858 & 1.114 & 0.010 & -1.044 & 0.039 & 12.668 & 0.048 \\
\hline & 49.90 & 0.600 & -27.23 & -1.03 & 1.860 & 1.122 & 0.009 & -1.060 & 0.033 & 11.909 & 0.042 \\
\hline & 74.78 & 0.600 & -29.23 & -0.89 & 1.865 & 1.136 & 0.008 & -1.105 & 0.026 & 11.276 & 0.035 \\
\hline \multirow[t]{6}{*}{24} & 5.04 & 0.600 & -23.40 & -1.25 & 1.854 & 1.103 & 0.011 & -1.002 & 0.046 & 13.453 & 0.055 \\
\hline & 24.78 & 0.600 & -25.23 & -1.18 & 1.854 & 1.103 & 0.011 & -0.995 & 0.043 & 12.720 & 0.052 \\
\hline & 49.90 & 0.600 & -27.42 & -1.04 & 1.857 & 1.111 & 0.009 & -1.019 & 0.036 & 11.950 & 0.045 \\
\hline & 74.91 & 0.600 & -29.31 & -0.89 & 1.864 & 1.133 & 0.008 & -1.092 & 0.027 & 11.286 & 0.036 \\
\hline & 100.12 & 0.600 & -30.97 & -0.74 & 1.873 & 1.161 & 0.006 & -1.184 & 0.020 & 10.710 & 0.029 \\
\hline & 125.06 & 0.600 & -32.24 & -0.65 & 1.884 & 1.198 & 0.006 & -1.293 & 0.015 & 10.209 & 0.024 \\
\hline \multirow[t]{6}{*}{25} & 5.08 & 1.001 & -2.35 & -0.46 & 1.250 & 1.377 & 0.033 & -1.040 & 0.061 & 13.441 & 0.071 \\
\hline & 24.89 & 1.001 & -2.66 & -0.46 & 1.248 & 1.354 & 0.031 & -0.995 & 0.058 & 12.744 & 0.068 \\
\hline & 49.92 & 1.001 & -2.80 & -0.39 & 1.251 & 1.391 & 0.027 & -1.066 & 0.049 & 11.927 & 0.059 \\
\hline & 74.92 & 1.001 & -2.93 & -0.33 & 1.254 & 1.425 & 0.024 & -1.127 & 0.043 & 11.271 & 0.053 \\
\hline & 100.07 & 1.000 & -3.00 & -0.27 & 1.257 & 1.464 & 0.022 & -1.197 & 0.038 & 10.712 & 0.048 \\
\hline & 125.07 & 1.001 & -2.87 & -0.21 & 1.261 & 1.528 & 0.020 & -1.313 & 0.034 & 10.196 & 0.044 \\
\hline \multirow[t]{6}{*}{26} & 5.03 & 1.001 & -2.35 & -0.46 & 1.250 & 1.377 & 0.033 & -1.040 & 0.061 & 13.443 & 0.071 \\
\hline & 24.83 & 1.001 & -2.63 & -0.45 & 1.249 & 1.361 & 0.031 & -1.010 & 0.058 & 12.731 & 0.068 \\
\hline & 49.90 & 1.001 & -2.79 & -0.39 & 1.251 & 1.394 & 0.027 & -1.070 & 0.049 & 11.924 & 0.059 \\
\hline & 74.81 & 1.000 & -2.89 & -0.32 & 1.254 & 1.433 & 0.024 & -1.142 & 0.042 & 11.259 & 0.052 \\
\hline & 100.09 & 1.000 & -3.00 & -0.27 & 1.257 & 1.464 & 0.022 & -1.197 & 0.038 & 10.712 & 0.048 \\
\hline & 125.04 & 1.001 & -2.90 & -0.22 & 1.261 & 1.523 & 0.020 & -1.303 & 0.034 & 10.206 & 0.044 \\
\hline
\end{tabular}

a See footnotes for Table 4.

constants. Although these ratios were typically $\sim 1: 18$ in experiments to measure $K_{m, 1 a}$, in experiments at ionic strengths of $0.3 \mathrm{~mol} \cdot \mathrm{kg}^{-1}$ the ratio was $\sim 1: 60$ (Tables 2 and 3). Sufficient acid was added to the corresponding test solutions to produce a formal concentration of 0.005 $\mathrm{mol} \cdot \mathrm{kg}^{-1}$ to $0.05 \mathrm{~mol} \cdot \mathrm{kg}^{-1}$ (Tables 2 and 3); the addition of acid was required to maintain sufficiently high degrees of association to permit accurate measurement of $\mathrm{K}_{\mathrm{m}, 1 \mathrm{la}}$.

The reference solutions used in experiments to measure $\mathrm{K}_{\mathrm{m}, 2 \mathrm{a}}$ had triflic acid to sodium triflate ratios 1:59 or less and were typically less than 1:119 (Table 3). The corresponding test solutions were prepared by combining the oxalic acid and sodium hydroxide stock solutions in a 2:3 molal ratio (Table 3 ).

Apparatus and Procedures. The modified hydrogenelectrode concentration cell used in these experiments has been described in detail previously (Mesmer et al., 1970; Wesolowski et al., 1984; Kettler et al., 1991; Palmer and Hyde, 1993) The solution compositions listed in Tables 2 and 3 refer to the cell configuration

$$
\text { Pt, } \mathrm{H}_{2}\left|\mathrm{H}_{2} \mathrm{C}_{2} \mathrm{O}_{4}, \mathrm{HX}, \mathrm{NaOH}, \mathrm{NaX}\right||\mathrm{HX}, \mathrm{NaX}| \mathrm{H}_{2}, \mathrm{Pt}
$$

where $\mathrm{X}$ refers to chloride or triflate. The experimental procedure described previously (Kettler et al., 1991) was used in this study with two modifications: (1) the porous Teflon liquid junction was saturated with reference solution prior to each experiment, and (2) the cell was allowed to equilibrate overnight at $5{ }^{\circ} \mathrm{C}$. The upper temperature limit $\left(125^{\circ} \mathrm{C}\right.$ ) was imposed by drifting potentials produced by decomposition of oxalic acid. Stable potentials were achieved at all temperatures reported in this study.

\section{Results}

The measured potentials (Tables 4-6) can be related to the molal concentration of hydrogen ions by the Nernst equation

$$
E=-(R T / F) \ln \left(\left[H^{+}\right]_{t} /\left[H^{+}\right]_{r}\right)-E_{l j}
$$


J ournal of Chemical and Engineering Data, Vol. 43, No. 3, 1998341

Table 6. Experimental Results of the Second Dissociation of Oxalic Acid in Aqueous Sodium Trifluoromethanesulfonate Mediaa

\begin{tabular}{|c|c|c|c|c|c|c|c|c|c|c|c|}
\hline expt & $\mathrm{t} /{ }^{\circ} \mathrm{C}$ & $\mathrm{I} /\left(\mathrm{mol} \cdot \mathrm{kg}^{-1}\right)$ & $\mathrm{E} \cdot 10^{3} \mathrm{~N}$ & $\mathrm{E}_{\mathrm{lj}} \cdot 10^{3} \mathrm{~N}$ & $\mathrm{pH}$ & $\bar{n}$ & $\sigma_{\bar{n}}$ & $\log K_{m, 2 a}$ & $\sigma_{2 a}$ & $\log K_{m, 2 b}$ & $\sigma_{2 \mathrm{~b}}$ \\
\hline \multirow[t]{7}{*}{6} & 5.03 & 0.100 & 77.56 & 3.97 & 3.777 & 0.492 & 0.006 & -3.760 & 0.011 & 10.772 & 0.017 \\
\hline & 24.93 & 0.100 & 86.49 & 4.21 & 3.833 & 0.494 & 0.006 & -3.821 & 0.011 & 9.971 & 0.017 \\
\hline & 49.87 & 0.100 & 101.22 & 4.06 & 3.942 & 0.498 & 0.006 & -3.936 & 0.011 & 9.125 & 0.017 \\
\hline & 49.87 & 0.100 & 101.32 & 4.06 & 3.944 & 0.498 & 0.006 & -3.937 & 0.011 & 9.122 & 0.017 \\
\hline & 75.06 & 0.100 & 119.87 & 3.59 & 4.086 & 0.501 & 0.006 & -4.086 & 0.011 & 8.391 & 0.017 \\
\hline & 100.10 & 0.100 & 141.60 & 3.03 & 4.252 & 0.503 & 0.006 & -4.256 & 0.011 & 7.755 & 0.017 \\
\hline & 125.02 & 0.100 & 166.43 & 2.94 & 4.443 & 0.505 & 0.006 & -4.450 & 0.011 & 7.187 & 0.017 \\
\hline \multirow[t]{3}{*}{8} & 5.06 & 0.100 & 78.77 & 3.98 & 3.799 & 0.485 & 0.006 & -3.769 & 0.011 & 10.761 & 0.017 \\
\hline & 24.95 & 0.100 & 87.58 & 4.22 & 3.851 & 0.487 & 0.006 & -3.826 & 0.011 & 9.965 & 0.017 \\
\hline & 100.05 & 0.100 & 142.69 & 3.03 & 4.267 & 0.495 & 0.006 & -4.257 & 0.011 & 7.755 & 0.017 \\
\hline \multirow[t]{6}{*}{27} & 5.08 & 0.100 & 54.85 & 1.28 & 3.795 & 0.484 & 0.006 & -3.764 & 0.011 & 10.767 & 0.017 \\
\hline & 24.93 & 0.100 & 61.57 & 1.62 & 3.847 & 0.486 & 0.006 & -3.819 & 0.011 & 9.974 & 0.017 \\
\hline & 49.84 & 0.100 & 73.57 & 1.69 & 3.952 & 0.489 & 0.006 & -3.931 & 0.011 & 9.131 & 0.017 \\
\hline & 74.99 & 0.100 & 89.13 & 1.50 & 4.090 & 0.492 & 0.006 & -4.074 & 0.011 & 8.406 & 0.017 \\
\hline & 100.08 & 0.100 & 108.12 & 1.21 & 4.254 & 0.494 & 0.006 & -4.243 & 0.011 & 7.771 & 0.017 \\
\hline & 124.98 & 0.100 & 130.52 & 1.26 & 4.446 & 0.496 & 0.006 & -4.438 & 0.011 & 7.203 & 0.017 \\
\hline \multirow[t]{6}{*}{28} & 5.02 & 0.100 & 39.19 & 0.56 & 3.799 & 0.484 & 0.006 & -3.769 & 0.011 & 10.763 & 0.017 \\
\hline & 24.88 & 0.100 & 44.44 & 0.93 & 3.847 & 0.486 & 0.006 & -3.819 & 0.011 & 9.974 & 0.017 \\
\hline & 49.88 & 0.100 & 54.86 & 1.07 & 3.952 & 0.489 & 0.006 & -3.930 & 0.011 & 9.129 & 0.017 \\
\hline & 74.75 & 0.100 & 68.85 & 0.96 & 4.090 & 0.492 & 0.006 & -4.074 & 0.011 & 8.409 & 0.017 \\
\hline & 100.08 & 0.100 & 86.50 & 0.75 & 4.257 & 0.494 & 0.006 & -4.246 & 0.011 & 7.766 & 0.017 \\
\hline & 125.01 & 0.100 & 107.31 & 0.83 & 4.447 & 0.496 & 0.006 & -4.440 & 0.011 & 7.198 & 0.017 \\
\hline \multirow[t]{6}{*}{44} & 4.99 & 1.000 & 66.02 & 0.41 & 3.514 & 0.506 & 0.006 & -3.520 & 0.011 & 10.964 & 0.021 \\
\hline & 24.99 & 1.000 & 71.91 & 0.45 & 3.533 & 0.507 & 0.006 & -3.541 & 0.011 & 10.193 & 0.021 \\
\hline & 49.97 & 1.000 & 82.38 & 0.45 & 3.602 & 0.510 & 0.006 & -3.615 & 0.011 & 9.375 & 0.021 \\
\hline & 74.93 & 1.000 & 95.73 & 0.39 & 3.702 & 0.513 & 0.006 & -3.721 & 0.011 & 8.675 & 0.021 \\
\hline & 100.09 & 1.000 & 111.78 & 0.32 & 3.824 & 0.517 & 0.006 & -3.849 & 0.011 & 8.059 & 0.021 \\
\hline & 125.01 & 1.001 & 130.18 & 0.32 & 3.961 & 0.519 & 0.006 & -3.992 & 0.011 & 7.517 & 0.021 \\
\hline \multirow[t]{6}{*}{45} & 5.08 & 0.600 & 55.87 & 0.39 & 3.552 & 0.495 & 0.006 & -3.539 & 0.011 & 10.913 & 0.020 \\
\hline & 25.06 & 0.600 & 61.49 & 0.43 & 3.579 & 0.497 & 0.006 & -3.571 & 0.011 & 10.134 & 0.020 \\
\hline & 49.89 & 0.600 & 71.57 & 0.43 & 3.656 & 0.502 & 0.006 & -3.656 & 0.011 & 9.312 & 0.020 \\
\hline & 74.98 & 0.600 & 84.79 & 0.38 & 3.765 & 0.507 & 0.006 & -3.775 & 0.011 & 8.600 & 0.020 \\
\hline & 100.09 & 0.600 & 100.94 & 0.31 & 3.900 & 0.513 & 0.006 & -3.919 & 0.011 & 7.975 & 0.020 \\
\hline & 124.99 & 0.601 & 119.55 & 0.31 & 4.049 & 0.517 & 0.006 & -4.076 & 0.011 & 7.426 & 0.020 \\
\hline \multirow[t]{6}{*}{46} & 5.09 & 0.273 & 41.92 & 0.35 & 3.640 & 0.498 & 0.006 & -3.633 & 0.011 & 10.836 & 0.017 \\
\hline & 24.94 & 0.273 & 47.09 & 0.48 & 3.679 & 0.500 & 0.006 & -3.675 & 0.011 & 10.053 & 0.017 \\
\hline & 49.91 & 0.273 & 56.80 & 0.52 & 3.769 & 0.504 & 0.006 & -3.772 & 0.011 & 9.218 & 0.017 \\
\hline & 74.87 & 0.273 & 69.92 & 0.47 & 3.894 & 0.508 & 0.006 & -3.905 & 0.011 & 8.501 & 0.017 \\
\hline & 100.06 & 0.273 & 86.08 & 0.38 & 4.042 & 0.512 & 0.006 & -4.060 & 0.011 & 7.870 & 0.017 \\
\hline & 125.00 & 0.273 & 105.23 & 0.40 & 4.211 & 0.515 & 0.006 & -4.234 & 0.011 & 7.312 & 0.017 \\
\hline \multirow[t]{6}{*}{47} & 5.05 & 0.600 & 68.35 & 0.69 & 3.552 & 0.495 & 0.006 & -3.540 & 0.011 & 10.914 & 0.020 \\
\hline & 24.95 & 0.600 & 74.96 & 0.72 & 3.581 & 0.497 & 0.006 & -3.573 & 0.011 & 10.137 & 0.020 \\
\hline & 49.97 & 0.600 & 86.29 & 0.69 & 3.658 & 0.502 & 0.006 & -3.658 & 0.011 & 9.309 & 0.020 \\
\hline & 75.06 & 0.600 & 100.72 & 0.60 & 3.767 & 0.508 & 0.006 & -3.778 & 0.011 & 8.597 & 0.020 \\
\hline & 100.08 & 0.600 & 117.81 & 0.51 & 3.899 & 0.512 & 0.006 & -3.918 & 0.011 & 7.977 & 0.020 \\
\hline & 125.01 & 0.601 & 137.72 & 0.49 & 4.050 & 0.517 & 0.006 & -4.076 & 0.011 & 7.426 & 0.020 \\
\hline \multirow[t]{6}{*}{48} & 5.13 & 0.316 & 73.81 & 1.38 & 3.641 & 0.478 & 0.006 & -3.599 & 0.011 & 10.860 & 0.020 \\
\hline & 24.94 & 0.316 & 81.21 & 1.39 & 3.675 & 0.482 & 0.006 & -3.641 & 0.011 & 10.079 & 0.020 \\
\hline & 50.01 & 0.316 & 93.66 & 1.30 & 3.760 & 0.490 & 0.006 & $\begin{array}{l}-3.739 \\
\end{array}$ & 0.011 & 9.240 & 0.020 \\
\hline & 74.94 & 0.316 & 109.18 & 1.14 & 3.876 & 0.498 & 0.006 & -3.871 & 0.011 & 8.524 & 0.020 \\
\hline & 100.07 & 0.316 & 127.83 & 0.97 & 4.018 & 0.506 & 0.006 & -4.027 & 0.011 & 7.892 & 0.020 \\
\hline & 125.02 & 0.316 & 149.32 & 0.92 & 4.180 & 0.513 & 0.006 & -4.200 & 0.011 & 7.333 & 0.020 \\
\hline
\end{tabular}

a See footnotes for Table 4.

where $\mathrm{E}$ is the measured potential, $\mathrm{R}$ is the gas constant, $\mathrm{F}$ is the Faraday constant, $\mathrm{T}$ is the thermodynamic temperature, and $\left[\mathrm{H}^{+}\right]_{\mathrm{t}}$ and $\left[\mathrm{H}^{+}\right]_{\mathrm{r}}$ refer to the molal concentration of hydrogen ions in the test and reference compartments, respectively. The $E_{l j}$ term designates the liquid junction potential. The value of $\left[\mathrm{H}^{+}\right]_{\mathrm{t}}$ can be calculated (Tables 4-6), given that the value of $\left[\mathrm{H}^{+}\right]_{r}$ is known. This simplified version of the Nernst equation requires the presence of matching ionic media on both sides of the liquid junction so that the calculated ratio of activity coefficients for $\mathrm{H}^{+}$in the test and reference compartments approaches unity. This equation is solved using an iterative process that considers partial vaporization of the solutions at elevated temperature, speciation of the test solution, and the consequent effects of vaporization and solution speciation on ionic strength and the liquid junction potential.
The liquid junction potential (Tables 4-6) is evaluated using the full Henderson approximation (eq 2-12 in Baes and Mesmer (1976)). Conductance data for $\mathrm{H}^{+}, \mathrm{OH}^{-}, \mathrm{Cl}^{-}$, $\mathrm{Na}^{+}, \mathrm{HSO}_{4}^{-}$, and $\mathrm{SO}_{4}^{2-}$ were obtained from Quist and Marshall (1965), whereas those for $\mathrm{CF}_{3} \mathrm{SO}_{3}^{-}$were obtained from $\mathrm{Ho}$ and Palmer (1995). The limiting molar conductances of bioxalate and oxalate were assumed to be identical to bisulfate and sulfate, respectively. Uncertainties introduced by this approximation are probably small: the conductances of oxalate and bioxalate at $25^{\circ} \mathrm{C}$ are within $25 \%$ of those for sulfate and bisulfate (Robinson and Stokes, 1959). The calculated liquid junction potential changes by less than $10 \%$ if the conductances assigned to bioxalate and oxalate are doubled. This value is well within the $25 \%$ error assigned to the liquid junction potential by using the Henderson equation (Mesmer and Holmes, 1992). 
342 J ournal of Chemical and Enginering Data, Vol. 43, No. 3, 1998

Table 7. Experimental Results for the First Dissociation of Oxalic Acid in Aqueous Sodium Chloride Media Published Previously (Kettler et al., 1991) and Recalculated as Part of This Studya

\begin{tabular}{|c|c|c|c|c|c|c|c|c|c|c|c|}
\hline exptl & $\mathrm{t} /{ }^{\circ} \mathrm{C}$ & $\mathrm{I} /\left(\mathrm{mol} \cdot \mathrm{kg}^{-1}\right)$ & $\mathrm{E} \cdot 10^{3} \mathrm{~N}$ & $E_{i j} \cdot 10^{3} \mathrm{~N}$ & $\mathrm{pH}$ & $\bar{n}$ & $\sigma_{\text {กิ }}$ & $\log K_{m, 1 a}$ & $\sigma_{1 a}$ & $\log K_{m, 1 b}$ & $\sigma_{1 \mathrm{~b}}$ \\
\hline \multirow[t]{5}{*}{19} & 0.00 & 4.998 & -27.30 & -0.32 & 1.490 & 1.417 & 0.008 & -1.351 & 0.012 & 13.893 & 0.042 \\
\hline & 24.88 & 4.999 & -31.00 & -0.30 & 1.471 & 1.345 & 0.007 & -1.203 & 0.012 & 13.084 & 0.042 \\
\hline & 49.85 & 5.000 & -34.18 & -0.28 & 1.462 & 1.314 & 0.007 & -1.135 & 0.011 & 12.380 & 0.041 \\
\hline & 74.81 & 5.001 & -36.93 & -0.25 & 1.461 & 1.311 & 0.006 & -1.127 & 0.010 & 11.739 & 0.040 \\
\hline & 99.79 & 5.003 & -39.49 & -0.23 & 1.463 & 1.318 & 0.006 & -1.143 & 0.009 & 11.160 & 0.039 \\
\hline \multirow[t]{5}{*}{20} & 24.86 & 2.001 & 25.83 & 1.89 & 1.452 & 1.234 & 0.041 & -0.957 & 0.089 & 12.863 & 0.105 \\
\hline & 49.83 & 2.002 & 27.55 & 1.68 & 1.439 & 1.181 & 0.036 & -0.808 & 0.092 & 12.255 & 0.108 \\
\hline & 74.82 & 2.002 & 30.23 & 1.51 & 1.443 & 1.197 & 0.031 & -0.851 & 0.075 & 11.596 & 0.091 \\
\hline & 99.79 & 2.002 & 33.06 & 1.35 & 1.448 & 1.218 & 0.027 & -0.909 & 0.061 & 11.023 & 0.077 \\
\hline & 124.77 & 2.003 & 36.16 & 1.27 & 1.457 & 1.255 & 0.024 & -1.002 & 0.050 & 10.493 & 0.066 \\
\hline \multirow[t]{4}{*}{21} & 49.85 & 0.101 & -25.88 & -5.67 & 1.792 & 1.187 & 0.066 & -1.174 & 0.178 & 11.864 & 0.184 \\
\hline & 74.83 & 0.101 & -27.90 & -5.16 & 1.805 & 1.212 & 0.051 & -1.248 & 0.125 & 11.210 & 0.131 \\
\hline & 99.79 & 0.100 & -29.49 & -4.62 & 1.823 & 1.243 & 0.038 & -1.339 & 0.087 & 10.655 & 0.093 \\
\hline & 124.77 & 0.099 & -30.54 & -4.18 & 1.844 & 1.280 & 0.030 & -1.438 & 0.063 & 10.182 & 0.069 \\
\hline \multirow[t]{4}{*}{22} & 49.84 & 3.172 & -56.23 & -0.72 & 1.310 & 1.329 & 0.032 & -1.010 & 0.060 & 12.214 & 0.076 \\
\hline & 74.77 & 3.173 & -60.44 & -0.64 & 1.314 & 1.361 & 0.028 & -1.075 & 0.050 & 11.520 & 0.066 \\
\hline & 99.80 & 3.173 & -64.69 & -0.56 & 1.317 & 1.386 & 0.024 & -1.123 & 0.042 & 10.937 & 0.058 \\
\hline & 124.77 & 3.175 & -68.78 & -0.52 & 1.321 & 1.421 & 0.022 & -1.188 & 0.037 & 10.411 & 0.053 \\
\hline \multirow[t]{4}{*}{23} & 49.85 & 1.030 & 2.74 & 0.22 & 1.321 & 1.270 & 0.022 & -0.901 & 0.043 & 12.063 & 0.053 \\
\hline & 74.82 & 1.030 & 3.14 & 0.21 & 1.323 & 1.294 & 0.020 & -0.953 & 0.039 & 11.409 & 0.049 \\
\hline & 99.79 & 1.030 & 3.67 & 0.19 & 1.327 & 1.335 & 0.019 & -1.036 & 0.035 & 10.830 & 0.045 \\
\hline & 124.77 & 1.030 & 4.34 & 0.20 & 1.332 & 1.392 & 0.018 & -1.146 & 0.033 & 10.308 & 0.043 \\
\hline \multirow[t]{4}{*}{24} & 49.89 & 0.332 & 0.23 & -0.22 & 1.767 & 1.138 & 0.007 & -1.009 & 0.013 & 11.955 & 0.022 \\
\hline & 74.82 & 0.332 & 0.94 & -0.18 & 1.778 & 1.159 & 0.007 & -1.080 & 0.012 & 11.296 & 0.021 \\
\hline & 99.79 & 0.331 & 2.02 & -0.15 & 1.792 & 1.186 & 0.007 & -1.169 & 0.013 & 10.731 & 0.022 \\
\hline & 124.77 & 0.331 & 3.52 & -0.09 & 1.810 & 1.220 & 0.007 & -1.270 & 0.014 & 10.240 & 0.023 \\
\hline \multirow[t]{4}{*}{25} & 49.86 & 1.982 & 16.42 & 1.21 & 1.264 & 1.262 & 0.040 & -0.826 & 0.086 & 12.234 & 0.096 \\
\hline & 74.82 & 1.982 & 18.08 & 1.09 & 1.267 & 1.279 & 0.035 & -0.864 & 0.072 & 11.581 & 0.082 \\
\hline & 99.79 & 1.982 & 19.90 & 0.98 & 1.271 & 1.307 & 0.031 & -0.925 & 0.060 & 11.005 & 0.070 \\
\hline & 124.77 & 1.982 & 21.97 & 0.93 & 1.279 & 1.357 & 0.028 & -1.028 & 0.051 & 10.467 & 0.061 \\
\hline \multirow[t]{6}{*}{26} & 0.00 & 3.172 & -47.28 & -0.91 & 1.310 & 1.325 & 0.045 & -1.001 & 0.086 & 13.948 & 0.102 \\
\hline & 24.93 & 3.172 & -51.44 & -0.79 & 1.316 & 1.376 & 0.036 & -1.104 & 0.065 & 12.882 & 0.081 \\
\hline & 49.89 & 3.172 & -56.19 & -0.72 & 1.311 & 1.335 & 0.032 & -1.024 & 0.060 & 12.200 & 0.076 \\
\hline & 74.82 & 3.172 & -60.26 & -0.64 & 1.317 & 1.385 & 0.027 & -1.121 & 0.048 & 11.474 & 0.064 \\
\hline & 99.80 & 3.173 & -64.55 & -0.56 & 1.319 & 1.403 & 0.024 & -1.154 & 0.041 & 10.906 & 0.057 \\
\hline & 124.78 & 3.175 & -68.76 & -0.52 & 1.321 & 1.423 & 0.022 & -1.192 & 0.037 & 10.407 & 0.053 \\
\hline \multirow[t]{5}{*}{27} & 24.92 & 4.002 & -32.77 & -0.33 & 1.537 & 1.230 & 0.008 & -1.033 & 0.014 & 13.085 & 0.044 \\
\hline & 49.89 & 4.002 & -35.57 & -0.30 & 1.537 & 1.231 & 0.008 & -1.034 & 0.012 & 12.317 & 0.042 \\
\hline & 74.86 & 4.003 & -38.25 & -0.27 & 1.539 & 1.238 & 0.007 & -1.051 & 0.010 & 11.662 & 0.040 \\
\hline & 99.81 & 4.004 & -40.67 & -0.24 & 1.544 & 1.257 & 0.006 & -1.100 & 0.009 & 11.067 & 0.039 \\
\hline & 124.77 & 4.007 & -42.69 & -0.22 & 1.553 & 1.293 & 0.006 & -1.185 & 0.008 & 10.505 & 0.038 \\
\hline \multirow[t]{5}{*}{28} & 24.86 & 3.002 & -33.69 & -0.35 & 1.646 & 1.165 & 0.009 & -0.981 & 0.013 & 12.981 & 0.029 \\
\hline & 49.93 & 3.002 & -36.60 & -0.32 & 1.646 & 1.164 & 0.008 & -0.978 & 0.013 & 12.219 & 0.029 \\
\hline & 74.84 & 3.003 & -39.25 & -0.28 & 1.649 & 1.178 & 0.007 & -1.017 & 0.011 & 11.554 & 0.027 \\
\hline & 99.80 & 3.004 & -41.81 & -0.25 & 1.653 & 1.195 & 0.007 & -1.064 & 0.009 & 10.976 & 0.025 \\
\hline & 124.78 & 3.006 & -44.27 & -0.23 & 1.658 & 1.214 & 0.006 & -1.114 & 0.008 & 10.468 & 0.024 \\
\hline
\end{tabular}

a See footnotes for Table 4.

The degree of association ( $\bar{n}$, Tables 4-6) is calculated from

$$
\begin{aligned}
& \bar{n}=\left(2 m\left(\mathrm{H}_{2} \mathrm{C}_{2} \mathrm{O}_{4}\right)-m(\right.\mathrm{NaOH})+\mathrm{m}(\mathrm{HX})- \\
& {\left.\left[\mathrm{H}^{+}\right]_{\mathrm{t}}+\left[\mathrm{OH}^{-}\right]_{\mathrm{t}}\right) / m\left(\mathrm{H}_{2} \mathrm{C}_{2} \mathrm{O}_{4}\right) }
\end{aligned}
$$

where $\mathrm{m}(\mathrm{X})$ denotes the stoichiometric molal concentrations of the designated species. For measurements of the first molal dissociation constant, the molal concentration of bioxalate was calculated from the proton balance expression

$$
\left[\mathrm{HC}_{2} \mathrm{O}_{4}^{-}\right]=\left(\left[\mathrm{H}^{+}\right]-\left[\mathrm{OH}^{-}\right]-\mathrm{m}(\mathrm{HX})\right) /\left(1+2 \mathrm{~K}_{\mathrm{m}, 2 \mathrm{a}} /\left[\mathrm{H}^{+}\right]\right)
$$

whereas the concentration of undissociated oxalic acid was calculated from mass balance considerations

$$
\left[\mathrm{H}_{2} \mathrm{C}_{2} \mathrm{O}_{4}\right]=\mathrm{m}\left(\mathrm{H}_{2} \mathrm{C}_{2} \mathrm{O}_{4}\right)-\left[\mathrm{HC}_{2} \mathrm{O}_{4}^{-}\right]\left(1+\mathrm{K}_{\mathrm{m}, 2 \mathrm{a}} /\left[\mathrm{H}^{+}\right]\right)
$$

The parallel equations for the second molal dissociation constant are

$$
\left[\mathrm{HC}_{2} \mathrm{O}_{4}^{-}\right]=\left(2 \mathrm{~m}\left(\mathrm{H}_{2} \mathrm{C}_{2} \mathrm{O}_{4}\right)-\mathrm{m}(\mathrm{NaOH})-\left[\mathrm{H}^{+}\right]+\left[\mathrm{OH}^{-}\right]\right) /
$$$$
\left(2\left[\mathrm{H}^{+}\right] / \mathrm{K}_{\mathrm{m}, 1 \mathrm{a}}+1\right)(9)
$$

and

$$
\left[\mathrm{C}_{2} \mathrm{O}_{4}^{2-}\right]=m\left(\mathrm{H}_{2} \mathrm{C}_{2} \mathrm{O}_{4}\right)-\left[\mathrm{HC}_{2} \mathrm{O}_{4}^{-}\right]\left(\left[\mathrm{H}^{+}\right] / \mathrm{K}_{\mathrm{m}, 1 \mathrm{a}}+1\right)
$$

Values of $K_{m, 1 a}$ and $K_{m, 2 a}$ (Tables 4-6) were then calculated using eqs 3 and 4.

\section{Discussion}

Fitting Process. In a number of previous reports (Kettler et al., 1991, 1992, 1995a; Bell et al., 1993; Bénézeth et al., 1997) from this laboratory, it has become apparent that the fitting process is simplified if the dissociation reactions are iso-Coulombic or pseudo-iso-Coulombic. This serves to minimize $\Delta z^{2}$ and hence reduce the degree of electrostriction (Lindsay, 1980). The Debye-Hückel limiting slope, changes in heat capacity, and changes in volume are thus minimized. This approach is achieved by recast- 
J ournal of Chemical and Engineering Data, Vol. 43, No. 3, 1998343

Table 8. Experimental Results of the Second Dissociation of Oxalic Acid in Aqueous Sodium Chloride Media Published Previously (Kettler et al., 1991) and Recalculated as Part of this Studya

\begin{tabular}{|c|c|c|c|c|c|c|c|c|c|c|c|}
\hline exptl & $\mathrm{t} /{ }^{\circ} \mathrm{C}$ & $\mathrm{I} /\left(\mathrm{mol} \cdot \mathrm{kg}^{-1}\right)$ & $\mathrm{E} \cdot 10^{3} \mathrm{~N}$ & $\mathrm{E}_{\mathrm{ij}} \cdot 10^{3} \mathrm{~N}$ & $\mathrm{pH}$ & $\bar{n}$ & $\sigma_{\bar{n}}$ & $\log K_{m, 2 a}$ & $\sigma_{2 \mathrm{a}}$ & $\log K_{m, 2 b}$ & $\sigma_{2 \mathrm{~b}}$ \\
\hline \multirow[t]{4}{*}{1} & 99.79 & 2.003 & 198.77 & 2.02 & 3.696 & 0.491 & 0.008 & -3.678 & 0.014 & 8.254 & 0.030 \\
\hline & 124.79 & 2.004 & 219.91 & 1.87 & 3.792 & 0.493 & 0.008 & -3.777 & 0.014 & 7.719 & 0.030 \\
\hline & 149.76 & 2.006 & 242.40 & 1.73 & 3.892 & 0.495 & 0.008 & -3.880 & 0.014 & 7.244 & 0.030 \\
\hline & 174.81 & 2.009 & 265.49 & 1.58 & 3.986 & 0.496 & 0.008 & -3.977 & 0.014 & 6.829 & 0.030 \\
\hline \multirow[t]{4}{*}{2} & 24.93 & 2.002 & 145.74 & 2.85 & 3.496 & 0.485 & 0.008 & -3.467 & 0.014 & 10.351 & 0.030 \\
\hline & 49.89 & 2.002 & 157.91 & 2.56 & 3.487 & 0.485 & 0.008 & -3.457 & 0.014 & 9.604 & 0.030 \\
\hline & 74.81 & 2.002 & 177.78 & 2.29 & 3.591 & 0.489 & 0.008 & -3.568 & 0.014 & 8.878 & 0.030 \\
\hline & 99.81 & 2.003 & 198.57 & 2.02 & 3.694 & 0.491 & 0.008 & -3.675 & 0.014 & 8.256 & 0.030 \\
\hline \multirow[t]{4}{*}{3} & 99.80 & 1.002 & 183.77 & 2.01 & 3.795 & 0.485 & 0.008 & -3.767 & 0.014 & 8.099 & 0.024 \\
\hline & 124.77 & 1.002 & 206.18 & 1.87 & 3.919 & 0.489 & 0.008 & -3.898 & 0.014 & 7.556 & 0.024 \\
\hline & 149.77 & 1.003 & 230.59 & 1.73 & 4.052 & 0.492 & 0.008 & -4.036 & 0.014 & 7.075 & 0.024 \\
\hline & 174.77 & 1.005 & 260.00 & 1.58 & 4.226 & 0.495 & 0.008 & -4.216 & 0.014 & 6.612 & 0.024 \\
\hline \multirow[t]{3}{*}{4} & 24.90 & 1.001 & 133.32 & 2.85 & 3.587 & 0.475 & 0.008 & -3.541 & 0.015 & 10.169 & 0.025 \\
\hline & 49.87 & 1.001 & 146.15 & 2.56 & 3.604 & 0.476 & 0.008 & -3.561 & 0.014 & 9.400 & 0.024 \\
\hline & 74.80 & 1.001 & 163.49 & 2.29 & 3.685 & 0.481 & 0.008 & -3.649 & 0.014 & 8.711 & 0.024 \\
\hline \multirow[t]{7}{*}{5} & 24.88 & 0.334 & 112.66 & 2.82 & 3.714 & 0.443 & 0.009 & -3.613 & 0.015 & 10.093 & 0.024 \\
\hline & 49.88 & 0.334 & 127.52 & 2.55 & 3.791 & 0.453 & 0.009 & -3.706 & 0.015 & 9.258 & 0.024 \\
\hline & 74.84 & 0.334 & 145.24 & 2.28 & 3.898 & 0.463 & 0.008 & -3.832 & 0.015 & 8.544 & 0.024 \\
\hline & 99.79 & 0.334 & 165.79 & 2.01 & 4.029 & 0.473 & 0.008 & -3.980 & 0.014 & 7.919 & 0.023 \\
\hline & 124.77 & 0.334 & 189.04 & 1.86 & 4.179 & 0.481 & 0.008 & -4.145 & 0.014 & 7.366 & 0.023 \\
\hline & 149.76 & 0.334 & 215.11 & 1.72 & 4.345 & 0.488 & 0.008 & -4.322 & 0.014 & 6.873 & 0.023 \\
\hline & 174.77 & 0.335 & 242.80 & 1.58 & 4.510 & 0.492 & 0.008 & -4.495 & 0.014 & 6.448 & 0.023 \\
\hline \multirow[t]{7}{*}{6} & 24.92 & 0.100 & 97.76 & 2.79 & 3.985 & 0.398 & 0.006 & -3.803 & 0.010 & 9.969 & 0.016 \\
\hline & 49.84 & 0.100 & 111.35 & 2.53 & 4.061 & 0.415 & 0.005 & -3.910 & 0.009 & 9.130 & 0.015 \\
\hline & 74.77 & 0.100 & 127.88 & 2.26 & 4.169 & 0.434 & 0.005 & -4.052 & 0.008 & 8.410 & 0.014 \\
\hline & 99.79 & 0.100 & 147.46 & 2.00 & 4.304 & 0.452 & 0.004 & -4.219 & 0.008 & 7.778 & 0.014 \\
\hline & 124.77 & 0.100 & 170.27 & 1.86 & 4.464 & 0.467 & 0.004 & -4.406 & 0.008 & 7.217 & 0.014 \\
\hline & 149.76 & 0.100 & 196.25 & 1.72 & 4.643 & 0.479 & 0.004 & -4.605 & 0.007 & 6.719 & 0.013 \\
\hline & 174.78 & 0.100 & 227.30 & 1.58 & 4.858 & 0.488 & 0.004 & -4.836 & 0.007 & 6.254 & 0.013 \\
\hline \multirow[t]{2}{*}{7} & 24.91 & 0.334 & 112.92 & 2.82 & 3.719 & 0.444 & 0.009 & -3.618 & 0.015 & 10.086 & 0.024 \\
\hline & 0.00 & 0.334 & 101.08 & 3.19 & 3.685 & 0.439 & 0.009 & -3.577 & 0.016 & 11.089 & 0.025 \\
\hline \multirow[t]{3}{*}{8} & 24.90 & 2.002 & 147.32 & 2.85 & 3.523 & 0.486 & 0.008 & -3.496 & 0.014 & 10.326 & 0.030 \\
\hline & 0.00 & 2.002 & 134.94 & 3.22 & 3.532 & 0.487 & 0.008 & -3.506 & 0.014 & 11.279 & 0.030 \\
\hline & 49.89 & 2.002 & 162.59 & 2.57 & 3.560 & 0.488 & 0.008 & -3.535 & 0.014 & 9.530 & 0.030 \\
\hline 9 & 24.91 & 1.001 & 133.93 & 2.85 & 3.597 & 0.476 & 0.008 & -3.553 & 0.015 & 10.159 & 0.025 \\
\hline 10 & 24.95 & 1.001 & 134.11 & 2.85 & 3.600 & 0.476 & 0.008 & -3.556 & 0.015 & 10.155 & 0.025 \\
\hline 11 & 74.80 & 5.002 & 117.10 & 0.07 & 3.697 & 0.484 & 0.008 & -3.665 & 0.014 & 9.202 & 0.044 \\
\hline \multirow[t]{6}{*}{12} & 49.87 & 5.001 & 109.40 & 0.08 & 3.708 & 0.484 & 0.008 & -3.677 & 0.014 & 9.837 & 0.044 \\
\hline & 74.82 & 5.002 & 117.13 & 0.07 & 3.697 & 0.484 & 0.008 & -3.665 & 0.014 & 9.201 & 0.044 \\
\hline & 99.79 & 5.00 & 125.63 & 0.06 & 3.698 & 0.484 & 0.008 & -3.6 & 0.014 & 8.637 & 0.044 \\
\hline & 124.77 & 5.006 & 135.79 & 0.05 & 3.720 & 0.485 & 0.008 & -3.690 & 0.014 & 8.114 & 0.044 \\
\hline & 149.77 & 5.01 & 146.22 & 0.05 & 3.742 & 0.486 & 0.008 & -3.713 & 0.014 & 7.642 & 0.044 \\
\hline & 174.77 & 5.02 & 157.82 & 0.04 & 3.775 & 0.487 & 0.008 & -3.749 & 0.014 & 7.202 & 0.044 \\
\hline \multirow[t]{6}{*}{13} & 49.89 & 3.149 & 89.16 & 0.08 & 3.593 & 0.463 & 0.008 & -3.526 & 0.014 & 9.694 & 0.030 \\
\hline & 74.82 & 3.150 & 98.18 & 0.07 & 3.624 & 0.466 & 0.008 & -3.562 & 0.014 & 9.030 & 0.030 \\
\hline & 99.83 & 3.151 & 108.83 & 0.06 & 3.672 & 0.470 & 0.008 & -3.617 & 0.014 & 8.440 & 0.030 \\
\hline & 124.83 & 3.1 & 120.77 & 0.0 & 3.730 & 0.4 & 0.00 & -3 & 0.0 & 7.913 & 0.030 \\
\hline & 149.76 & 3.156 & 134.39 & 0.05 & 3.802 & 0.47 & 0.008 & -3.762 & 0.014 & 7.432 & 0.030 \\
\hline & 174.78 & 3.161 & 149.50 & 0.04 & 3.882 & 0.483 & 0.008 & -3.850 & 0.014 & 6.991 & 0.030 \\
\hline \multirow[t]{3}{*}{14} & 24.89 & 5.000 & 103.99 & 0.09 & 3.760 & 0.486 & 0.008 & -3.733 & 0.014 & 10.554 & 0.044 \\
\hline & 0.00 & 5.0 & 100.41 & 0.11 & 3.855 & 0.490 & 0.008 & -3.8 & 0.014 & 11.411 & 0.044 \\
\hline & 49.85 & 5.001 & 109.79 & 0.08 & 3.714 & 0.484 & 0.008 & -3.684 & 0.014 & 9.831 & 0.044 \\
\hline 15 & 24.92 & 3.149 & 81.80 & 0.09 & 3.586 & 0.462 & 0.008 & -3.517 & 0.014 & 10.463 & 0.030 \\
\hline & 0.00 & 3.149 & 76.60 & 0.11 & 3.616 & 0.465 & 0.008 & -3.553 & 0.014 & 11.389 & 0.030 \\
\hline & 49.87 & 3.14 & 89.32 & 0.08 & 3.55 & 0.463 & 0.008 & -3.529 & 0.014 & 9.689 & 0.030 \\
\hline & 74.83 & 3.150 & 98.88 & 0.07 & 3.634 & 0.467 & 0.008 & -3.573 & 0.014 & 9.016 & 0.030 \\
\hline 16 & & 0.185 & 93.55 & 3.22 & 3.803 & 0.417 & 0.005 & -3.655 & 0.010 & 11.034 & 0.016 \\
\hline 10 & 24.93 & 0.185 & 104.95 & 2.86 & 3.841 & 0.424 & 0.005 & -3.706 & 0.009 & 10.027 & 0.015 \\
\hline & 49.87 & 0.185 & 118.94 & 2.60 & 3.914 & 0.436 & 0.0 & -3.801 & 0.008 & 9.196 & 0.014 \\
\hline & 74.86 & 0.185 & 136.41 & 2.33 & 4.027 & 0.451 & 0.004 & -3.940 & 0.008 & 8.472 & 0.014 \\
\hline & 99.77 & 0.185 & 157.42 & 2.07 & 4.173 & 0.466 & 0.004 & -4.112 & 0.008 & 7.831 & 0.014 \\
\hline & 124.77 & 0.185 & 180.86 & 1.94 & 4.333 & 0.477 & 0.004 & -4.291 & 0.007 & 7.271 & 0.013 \\
\hline & 149.77 & 0.185 & 208.22 & 1.80 & 4.520 & 0.486 & 0.004 & -4.494 & 0.007 & 6.760 & 0.013 \\
\hline & & 18 & 24166 & 166 & 4775 & 0.493 & & -47 & & & \\
\hline
\end{tabular}

a See footnotes for Table 4.

ing eqs 1 and 2 in the base or anionic forms

for which the molal dissociation constants are defined by

$$
\mathrm{H}_{2} \mathrm{C}_{2} \mathrm{O}_{4}+\mathrm{OH}^{-}=\mathrm{HC}_{2} \mathrm{O}_{4}^{-}+\mathrm{H}_{2} \mathrm{O}
$$

$$
\mathrm{K}_{\mathrm{m}, 1 \mathrm{~b}}=\left[\mathrm{HC}_{2} \mathrm{O}_{4}^{-}\right] /\left(\left[\mathrm{H}_{2} \mathrm{C}_{2} \mathrm{O}_{4}\right]\left[\mathrm{OH}^{-}\right]\right)
$$

and

and

$$
\mathrm{HC}_{2} \mathrm{O}_{4}^{-}+\mathrm{OH}^{-}=\mathrm{C}_{2} \mathrm{O}_{4}^{2-}+\mathrm{H}_{2} \mathrm{O}
$$

$$
\mathrm{K}_{\mathrm{m}, 2 \mathrm{~b}}=\left[\mathrm{C}_{2} \mathrm{O}_{4}^{2-}\right] /\left(\left[\mathrm{HC}_{2} \mathrm{O}_{4}^{-}\right]\left[\mathrm{OH}^{-}\right]\right)
$$


344 J ournal of Chemical and Enginering Data, Vol. 43, No. 3, 1998

Table 9. Values Obtained for Parameters for Eq 17

\begin{tabular}{lccccc}
\hline term & Harned and Fallon & Pinching and Bates 1-4 & Pinching and Bates 5 & Pinching and Bates 6 & Pinching and Bates 7 \\
\hline $\mathrm{p}_{1}$ & -1.314325 & -1.310412 & -1.290979 & -1.324607 & -1.310050 \\
$\mathrm{p}_{2}$ & 3293.019 & 3288.747 & 3285.281 & 3294.901 & 3290.594 \\
$\mathrm{p}_{3}$ & 0.05158081 & 0.08137941 & 0.02871844 & 0.02173294 & 0.06509261 \\
$\mathrm{p}_{4}$ & 0.5263629 & 0.1017003 & 0.1248080 & 0.1412951 & 0.1556312 \\
$\mathrm{AF}^{\mathrm{a}}$ & 0.2346 & 0.3905 & 0.2561 & 0.3387 & 0.2595
\end{tabular}

a Agreement factor (eq 18).

The values for $\log K_{m, n a}$ are converted to the respective values for $\log K_{m, n b}$ by the subtraction of the appropriate values for $\log \mathrm{K}_{m, w}$ as measured in Na-triflate (Palmer and Drummond, 1988) or $\mathrm{NaCl}$ media (Busey and Mesmer, 1978). The standard equilibrium constants for eqs 11 and 12 are designated by $\mathrm{K}_{1 \mathrm{~b}}^{\ominus}$ and $\mathrm{K}_{2 \mathrm{~b}}^{\ominus}$, respectively, are defined in the usual way (Mills et al., 1993), and are equal to the products of the equilibrium activities of the species listed in eqs 11 and 12 raised to the respective stoichiometric numbers.

The ORGLS (Busing and Levy, 1963) general leastsquares routine was used to perform a weighted regression of the data. The fitting process was an iterative one because the solution speciation can only be calculated if one of the molal dissociation constants is known and because five separate data sets were used in the fitting process. These data sets comprised measurements of (1) $\mathrm{K}_{\mathrm{m}, 1 \mathrm{~b}}$ and $\mathrm{K}_{\mathrm{m}, 2 \mathrm{~b}}$ made in $\mathrm{Na}$-triflate media (Tables 5 and 6), (2) $\mathrm{K}_{\mathrm{m}, 1 \mathrm{~b}}$ made in $\mathrm{NaCl}$ media (Table 4), (3) $\mathrm{K}_{\mathrm{m}, 1 \mathrm{~b}}$ and $\mathrm{K}_{\mathrm{m}, 2 \mathrm{~b}}$ in $\mathrm{NaCl}$ reported by Kettler et al. (1991) (Tables 7 and 8), (4) $K_{m, 2 b}$ made by Harned and Fallon (1939), and (5) $K_{m, 2 b}$ made by Pinching and Bates (1948). The latter two sets of measurements were made using $\mathrm{H}$ arned cells.

Several of the data sets that were used in the previous fitting process (Kettler et al., 1991) have been omitted here. Although the glass electrode measurements of Kurz and Farrar (1969) (Table 1) were utilized previously, those authors did not publish sufficient data to permit recalculation of the values of $\mathrm{K}_{1 a}^{\ominus}$ reported originally. The titration cal orimetry measurements of Christensen et al. (1967) were not reported in sufficient detail to permit recalculation of $\Delta \mathrm{H}$ on the basis of the results of this study. Measurements by Parton and Gibbons (1939) using a cell without a liquid junction were limited to a few values collected at $25^{\circ} \mathrm{C}$ (Table 1), and although McAuley and Nancollas (1961) reported results obtained from $\mathrm{H}$ arned cell measurements at five temperatures from $0{ }^{\circ} \mathrm{C}$ to $45^{\circ} \mathrm{C}$, solution compositions and potentials were presented only for the measurements at $25^{\circ} \mathrm{C}$.

The fitting process involved (1) calculation of values for $\mathrm{K}_{\mathrm{m}, 2 \mathrm{~b}}$ using the most recent equations describing $\mathrm{K}_{\mathrm{m}, 1 \mathrm{~b}},(2)$ extrapolation of the Harned and Fallon (1939) and Pinching and Bates (1948) data to infinite dilution to obtain values of $\mathrm{K}_{2 \mathrm{~b}}^{\ominus}$ at temperatures from $0{ }^{\circ} \mathrm{C}$ to $50{ }^{\circ} \mathrm{C}$, (3) fitting the data for $\mathrm{K}_{\mathrm{m}, 2 \mathrm{~b}}$ collected in $\mathrm{NaCl}$ and $\mathrm{Na}$-triflate media simultaneously with the values for $\mathrm{K}_{2 \mathrm{~b}}^{\ominus}$ obtained in the previous step, (4) calculation of values for $K_{m, 1 b}$ using the models devel oped for $\mathrm{K}_{\mathrm{m}, 2 \mathrm{~b}}$ in $\mathrm{NaCl}$ and $\mathrm{Na}$-triflate media, and (5) performing a simultaneous fit of the data for $K_{m, 1 b}$ in $\mathrm{NaCl}$ and $\mathrm{Na}$-triflate media. This process was repeated until the values of $K_{m, 1 b}$ and $K_{m, 2 b}$ obtained in consecutive iterations agreed to within 0.0001 log units.

Recalculation of Data Published Previously. Because the fitting process is iterative, the experimental values of $K_{m, n a}$ and $K_{m, n b}$ obtained in our previous study (Kettler et al., 1991) have been recalculated using eqs 9-14 and are presented in Tables 7 and 8 . The Harned cell data (Harned and Fallon, 1939; Pinching and Bates, 1948) are well-documented and recognized to be of high quality and should therefore be included in the fitting process. To ensure consistency with the data presented in this study, particularly with regard to calculation of activity coefficients, the Harned cell data have been recalculated using the Nernst equation

$$
\mathrm{E}=\mathrm{E}^{\circ}-\mathrm{RT} / \mathrm{F}\left(\operatorname{In}\left[\mathrm{Cl}^{-}\right]\left[\mathrm{H}^{+}\right]+\ln \gamma\left(\mathrm{Cl}^{-}\right) \gamma\left(\mathrm{H}^{+}\right)\right)
$$

where $\gamma(X)$ refers to the activity coefficient of the designated ion. Values for the standard potential $\left(\mathrm{E}^{\circ}\right)$ of the $\mathrm{AgCl}$ electrode have been taken from Bates (1964). The activity coefficients are calculated using the extended Debye-Hückel equation of Pitzer (1973) where

$$
\begin{aligned}
& \ln \gamma\left(\mathrm{H}^{+}\right) \gamma\left(\mathrm{Cl}^{-}\right)= \\
& 2 \mathrm{~A}_{\phi}\left(\left(\mathrm{I} / \mathrm{kg} \cdot \mathrm{mol}^{-1}\right)^{0.5} /\left(1+1.2\left(\mathrm{I} / \mathrm{kg} \cdot \mathrm{mol}^{-1}\right)^{0.5}\right)+\right. \\
& \left.(2 / 1.2) \ln \left(1+1.2\left(\mathrm{I} / \mathrm{kg} \cdot \mathrm{mol}^{-1}\right)^{0.5}\right)\right)
\end{aligned}
$$

and $\mathrm{A}_{\phi}$ is the Debye-Hückel osmotic coefficient parameter (calculated using eq 9 in Dickson et al. (1990)), whereas the ionic strength is represented by I. After eq 15 was solved for $\left[\mathrm{H}^{+}\right]$, the oxalate species were distributed according to eqs $11-14$ and $K_{m, 2 a}$ was calculated from $K_{m, 2 b}$ using the molal dissociation constant of water (Busey and Mesmer, 1978).

Fitting of Harned Cell Data. The Harned and Fallon (1939) data set comprises 87 measurements made at 11 different temperatures from $0{ }^{\circ} \mathrm{C}$ to $50^{\circ} \mathrm{C}$ in 8 different solutions with ionic strengths ranging from $\sim 0.03 \mathrm{~mol} \cdot \mathrm{kg}^{-1}$ to $\sim 0.15 \mathrm{~mol} \cdot \mathrm{kg}^{-1}$. Each solution had the same ratio of oxalate to chloride (2:1), so that all of the data could be treated as a single set. Regression of the $\log K_{m, 2 b}$ values as a function of temperature and ionic strength yielded

$$
\begin{gathered}
\log K_{m, 2 b}=2\left(A_{\phi}\left((I(\mathrm{~kg} / \mathrm{mol}))^{0.5}\right) /\left(1+1.2(I(\mathrm{~kg} / \mathrm{mol}))^{0.5}\right)+\right. \\
\left.(2 / 1.2) \ln \left(1+1.2(\mathrm{I}(\mathrm{kg} / \mathrm{mol}))^{0.5}\right) / / \mathrm{ln} 10\right)+ \\
\mathrm{p}_{1}+\mathrm{p}_{2}(\mathrm{~K} / \mathrm{T})+\mathrm{p}_{3} \mathrm{f}(\mathrm{I})+\mathrm{p}_{4}(\mathrm{I}(\mathrm{kg} / \mathrm{mol}))^{2}-\log \mathrm{a}_{\mathrm{w}}(17)
\end{gathered}
$$

where

$$
f(I)=1-\left(1+2(I(\mathrm{~kg} / \mathrm{mol}))^{0.5}\right) \exp \left(-2(\mathrm{I}(\mathrm{kg} / \mathrm{mol}))^{0.5}\right)
$$

and $a_{w}$ is the activity of water (Busey and Mesmer, 1976). The first term in eq 17 defines the extended Debye-Hückel limiting slope (Pitzer, 1973), whereas parameters $p_{1}$ and $p_{2}$ define $\log K_{2 b}^{\ominus}$ and the remaining quantify the deviation from the limiting law. The values of parameters $p_{1}-$ $p_{4}$ are shown in Table 9 . The goodness of fit is quantified using the agreement factor

$$
A F=\left(\left(\sum W_{i}\left(\log K_{m, i(o b s)}-\log K_{m, i(\text { calc })}\right)^{2}\right) /\left(N-N_{v}\right)\right)_{(19)}^{0.5}
$$

where $\mathrm{W}$ is the squared reciprocal of the estimated error assigned to any individual determination of $\log \mathrm{K}_{\mathrm{m}}, \mathrm{N}$ is 
Table 10. Values of $\mathrm{pK}_{2 a}^{\ominus}$ for Oxalic Acid Obtained by Recalculating the Data of Harned and Fallon (1939) and Pinching and Bates (1948)

\begin{tabular}{|c|c|c|c|c|c|}
\hline $\mathrm{t} /{ }^{\circ} \mathrm{C}$ & Harned and Fallon & $\begin{array}{c}\text { Pinching and Bates } \\
\text { series } 1-4\end{array}$ & $\begin{array}{c}\text { Pinching and Bates } \\
\text { series } 5\end{array}$ & $\begin{array}{c}\text { Pinching and Bates } \\
\text { series } 6\end{array}$ & $\begin{array}{c}\text { Pinching and Bates } \\
\text { series } 7\end{array}$ \\
\hline 0 & $4.200 \pm 0.004$ & $4.211 \pm 0.002$ & $4.204 \pm 0.013$ & $4.203 \pm 0.004$ & $4.204 \pm 0.004$ \\
\hline 5 & $4.203 \pm 0.004$ & $4.215 \pm 0.002$ & $4.208 \pm 0.013$ & $4.207 \pm 0.004$ & $4.208 \pm 0.004$ \\
\hline 10 & $4.212 \pm 0.004$ & $4.223 \pm 0.002$ & $4.216 \pm 0.013$ & $4.216 \pm 0.004$ & $4.217 \pm 0.004$ \\
\hline 15 & $4.226 \pm 0.004$ & $4.237 \pm 0.002$ & $4.230 \pm 0.013$ & $4.230 \pm 0.004$ & $4.230 \pm 0.004$ \\
\hline 20 & $4.242 \pm 0.004$ & $4.252 \pm 0.002$ & $4.245 \pm 0.013$ & $4.246 \pm 0.004$ & $4.246 \pm 0.004$ \\
\hline 25 & $4.262 \pm 0.004$ & $4.273 \pm 0.002$ & $4.265 \pm 0.013$ & $4.266 \pm 0.004$ & $4.266 \pm 0.004$ \\
\hline 30 & $4.285 \pm 0.004$ & $4.295 \pm 0.002$ & $4.287 \pm 0.013$ & $4.289 \pm 0.004$ & $4.288 \pm 0.004$ \\
\hline 35 & $4.310 \pm 0.004$ & $4.320 \pm 0.002$ & $4.312 \pm 0.013$ & $4.314 \pm 0.004$ & $4.313 \pm 0.004$ \\
\hline 40 & $4.336 \pm 0.004$ & $4.346 \pm 0.002$ & $4.338 \pm 0.013$ & $4.341 \pm 0.004$ & $4.340 \pm 0.004$ \\
\hline 45 & $4.366 \pm 0.004$ & $4.375 \pm 0.002$ & $4.367 \pm 0.013$ & $4.370 \pm 0.004$ & $4.369 \pm 0.004$ \\
\hline 50 & $4.396 \pm 0.004$ & $4.405 \pm 0.002$ & $4.396 \pm 0.013$ & $4.400 \pm 0.004$ & $4.399 \pm 0.004$ \\
\hline
\end{tabular}

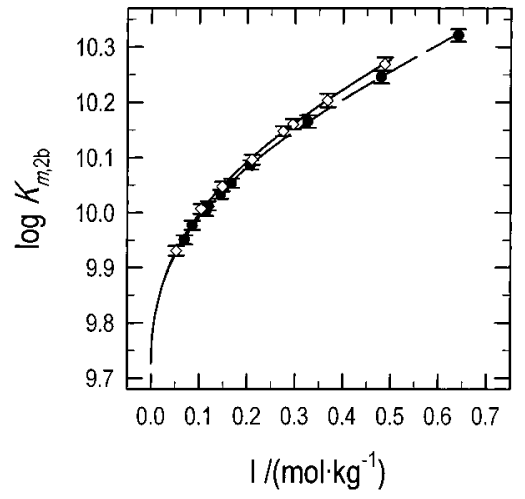

Figure 1. Comparisons of Harned cell data obtained in two different ionic media by Pinching and Bates (1948) at $25^{\circ} \mathrm{C}$. The filled circles $(\bullet)$ and dashed line designate the results from series 6 measurements and the resulting regression equation (eq 17), respectively, whereas the open diamonds $(\diamond)$ and solid line designate the results from series 7 .

the number of observations, and $N_{v}$ is the number of independent variables in the fit. The agreement factor for the Harned and Fallon (1939) regression is 0.23 and indicates that the actual scatter around eq 17 is 0.23 times the anticipated scatter using the uncertainties in solution composition and potentials given by Harned and Fallon. The values of $\mathrm{K}_{2 \mathrm{a}}^{\ominus}$ derived from this regression after multiplication by the standard dissociation constant of water (Busey and Mesmer, 1978) differ from the values originally reported by Harned and Fallon (1939) by ca. -0.03 log units (cf. Table 10 with Table 1).

The data collected by Pinching and Bates (1948) comprised 617 measurements made at 11 temperatures in 4 different ionic media. These experiments were classified into seven different series. Series 1-4 were performed in solutions in which the ratio of total oxalate to chloride was 2.4:1. Series 5 was performed with solutions in which this ratio was $6: 1$, whereas the ratios for series 6 and 7 were $10: 1$ and 2:1, respectively. The values obtained are sufficiently different in the various ionic media (Figure 1) that four different extrapolations to infinite dilution were performed to correspond to the different ionic media. The best fit in each case was provided by eq 17 using the parameters listed in Table 9. The values of $\mathrm{pK}_{2 \mathrm{a}}^{\ominus}$ obtained from the series 5-7 data were in excellent agreement with the values obtained by recalculation and regression of the Harned and Fallon (1939) data (Table 10). The values of $\mathrm{pK}_{2 \mathrm{a}}^{\ominus}$ obtained from the series 1-4 data are ca. $0.01 \mathrm{log}$ units greater than those reported in the original study. The overall effect is that the difference between the values of $\mathrm{pK}_{2 \mathrm{a}}^{\ominus}$ obtained from these two groups decreases from ca. $0.025 \log$ units to $0.01 \log$ unit (cf. Table 10 with Table 1).

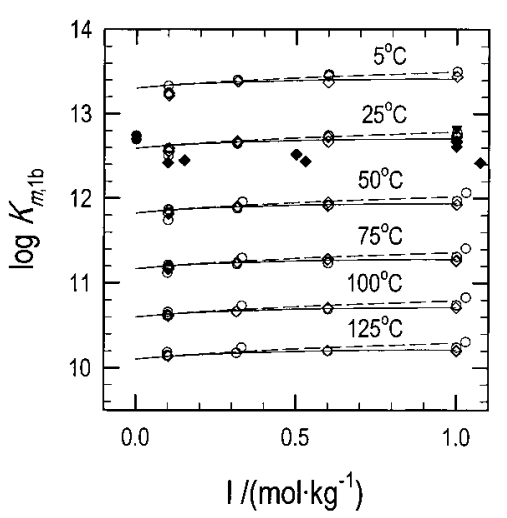

Figure 2. Relation between $\log K_{m, 1 b}$ and solution ionic strength to $1 \mathrm{~mol} \cdot \mathrm{kg}^{-1}$. The dashed curves are generated from eq 20 when solved for $\mathrm{NaCl}$ media, whereas the solid curves are generated when eq 20 is solved for $\mathrm{Na}$-triflate media. The symbols represent measurements made in $\mathrm{NaCl}$ media reported in Tables 4 and 6 $(\mathrm{O})$, measurements made in Na-triflate media reported in Table 5 $(\diamond)$, the $25^{\circ} \mathrm{C}$ measurements reported by McAuley and Nancollas (1961) and Parton and Gibbons (1939), (•), the value reported by Cruywagen et al. (1986) ( $\mathbf{\nabla})$, and measurements made in $\mathrm{NaClO}_{4}$ and $\mathrm{KNO}_{3}$ media listed in Table $1(\bullet)$. Measurements reported as $\mathrm{K}_{\mathrm{m}, 1 \mathrm{a}}$ in $\mathrm{NaClO}_{4}$ and $\mathrm{KNO}_{3}$ solutions were converted to $\mathrm{K}_{\mathrm{m}, 1 \mathrm{~b}}$ using values for $\mathrm{K}_{\mathrm{m}, \mathrm{w}}$ in $\mathrm{NaClO}_{4}$ and $\mathrm{KNO}_{3}$ solutions as reported by Maeda et al. (1987).

Fitting of the First Molal Dissociation Constant of Oxalic Acid. The fitting process for the first dissociation of oxalic acid in the base form (eq 13) utilized only those data in Tables 4, 5, and 7. The data, which were obtained in $\mathrm{NaCl}$ and $\mathrm{Na}$-triflate media, were fitted simultaneously yielding a five-parameter function of temperature and ionic strength

$$
\begin{aligned}
\log K_{m, 1 b}=p_{1}+p_{2}(K / T)+ & p_{3} f(I)+p_{4} I(k g / m o l)+ \\
& p_{5}(I(k g / m o l))^{2}-\log a_{w}
\end{aligned}
$$

where $p_{1}$ and $p_{2}$ define the logarithm of the standard equilibrium constant $\left(\mathrm{K}_{1 \mathrm{~b}}^{\ominus}\right), \mathrm{p}_{3}$ applies to both $\mathrm{NaCl}$ and $\mathrm{Na}$-triflate media, $\mathrm{p}_{4}$ is used only in $\mathrm{NaCl}$ media, and $\mathrm{p}_{5}$ applies only to $\mathrm{Na}$-triflate solutions. The values determined for the five parameters are $p_{1}=2.68385, p_{2}=$ 2954.02, $p_{3}=0.234331, p_{4}=0.0404618$, and $p_{5}=$ -0.0418473 . This function provides an excellent fit to data in both $\mathrm{NaCl}$ and $\mathrm{Na}$-triflate media (Figures 2 and 3 ); the agreement factor for this model is 0.79 , and the residuals of the regression are distributed randomly when plotted against ionic strength and temperature (Figure 4). Values of $\log K_{m, 1 a}$ obtained by adding the results of eq 20 to the logarithm of molal dissociation constant of water (Palmer and Drummond, 1988; Busey and Mesmer, 1978) are presented in Tables 11 and 12. Although eq 20 is 


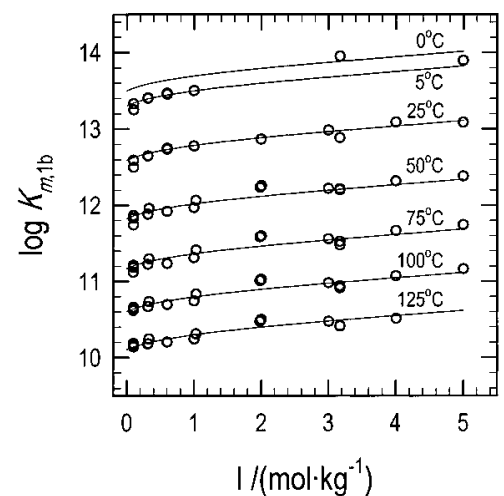

Figure 3. Relation between $\log K_{m, 1 b}$ and solution ionic strength to $5 \mathrm{~mol} \cdot \mathrm{kg}^{-1}$. The lines are generated by solving eq 20 for $\mathrm{NaCl}$ media. The symbols represent the measurements made in $\mathrm{NaCl}$ media reported in Tables 4 and 7.
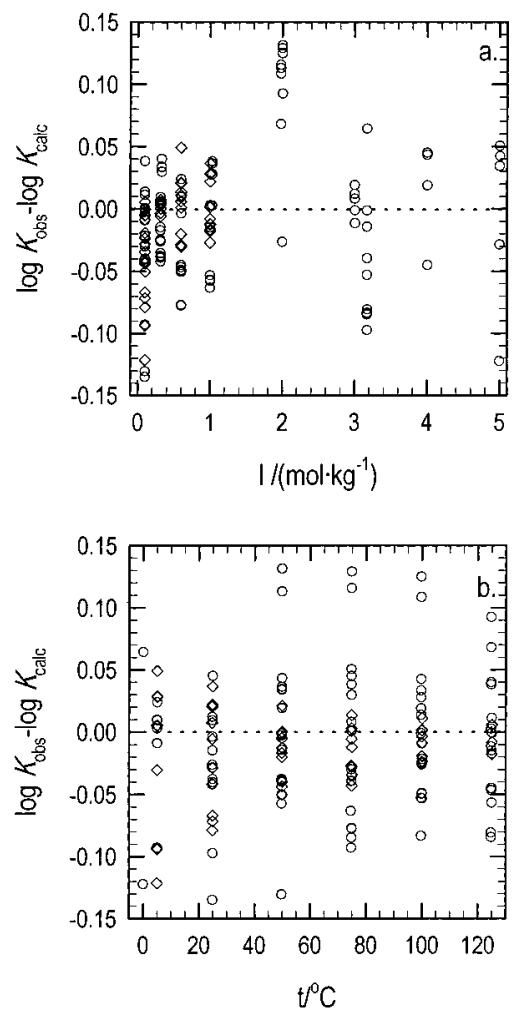

Figure 4. Deviation plots of the difference between experimentally determined values of $\log K_{m, 1 b}$ presented in Tables 4 and 7 and eq 20 as a function of (a) ionic strength and (b) temperature. Symbols designate measurements made in $\mathrm{NaCl}$ media $(O$, Tables 4 and 7) and Na-triflate media ( $\diamond$, Table 5).

strictly valid only to I $=1 \mathrm{~mol} \cdot \mathrm{kg}^{-1}$ in Na-triflate media, it applies up to I $=5 \mathrm{~mol} \cdot \mathrm{kg}^{-1}$ in NaCl media and is valid from $0{ }^{\circ} \mathrm{C}$ to $125^{\circ} \mathrm{C}$ in both media.

The model presented in eq 20 is in good agreement with a value obtained by Cruywagen et al. (1986) at $25^{\circ} \mathrm{C}$ in 1 $\mathrm{mol} \cdot \mathrm{kg}^{-1} \mathrm{NaCl}$ solution (Figure 2). On the other hand, measurements made in $\mathrm{NaClO}_{4}$ and $\mathrm{KNO}_{3}$ media differ significantly from those reported here (Figures 2 and 3), and the media-dependent variations noted in the first paper (Kettler et al., 1991) are preserved in these data. A potentially greater problem results from the fact that the value of $\mathrm{pK}_{1 \mathrm{a}, 298}^{\ominus}$ obtained from eq 20 is in poor agreement with the generally accepted value of $\mathrm{pK}_{1 \mathrm{l}, 298}^{\ominus}$ obtained by McAuley and Nancollas (1961) and the similar value reported by Parton and Gibbons (1939) (Table 1). These two data sets were recalculated to better compare them
Table 11. Summary of Thermodynamic Quantities for the First Dissociation of Oxalic Acid in Aqueous Sodium Chloride Media at the Saturation Vapor Pressure ${ }^{a}$

\begin{tabular}{|c|c|c|c|c|}
\hline${ }^{\circ} \mathrm{C}$ & $\log K_{m, 1 a}$ & $\begin{array}{c}\Delta \mathrm{H}_{\mathrm{la}} / \\
\left(\mathrm{kJ} \cdot \mathrm{mol}^{-1}\right)\end{array}$ & $\begin{array}{c}\Delta \mathrm{S}_{1 \mathrm{a}} / \\
\left(\mathrm{J} \cdot \mathrm{mol}^{-1} \cdot \mathrm{K}^{-1}\right)\end{array}$ & $\begin{array}{c}\Delta \mathrm{C}_{\mathrm{p}, 1 \mathrm{al}} / \\
\left(\mathrm{J} \cdot \mathrm{mol}^{-1} \cdot \mathrm{K}^{-1}\right)\end{array}$ \\
\hline $\begin{array}{r}0 \\
25 \\
50 \\
75 \\
100 \\
125\end{array}$ & $\begin{array}{l}-1.443 \pm 0.057 \\
-1.401 \pm 0.052 \\
-1.447 \pm 0.047 \\
-1.540 \pm 0.046 \\
-1.664 \pm 0.050 \\
-1.811 \pm 0.051\end{array}$ & $\begin{array}{r}I=0 \mathrm{~mol} \cdot \\
6.0 \pm 0.9 \\
-0.7 \pm 0.7 \\
-5.9 \pm 0.8 \\
-10.3 \pm 0.9 \\
-14.5 \pm 1.0 \\
-19.1 \pm 1.0\end{array}$ & $\begin{array}{r}\mathrm{kg}^{-1} \\
-6 \pm 3 \\
-29 \pm 2 \\
-46 \pm 2 \\
-59 \pm 3 \\
-71 \pm 3 \\
-83 \pm 3\end{array}$ & $\begin{array}{l}-317 \pm 18 \\
-231 \pm 6 \\
-186 \pm 5 \\
-169 \pm 5 \\
-174 \pm 5 \\
-197 \pm 5\end{array}$ \\
\hline $\begin{array}{r}0 \\
25 \\
50 \\
75 \\
100 \\
125\end{array}$ & $\begin{array}{l}-1.203 \pm 0.044 \\
-1.152 \pm 0.038 \\
-1.184 \pm 0.035 \\
-1.262 \pm 0.034 \\
-1.367 \pm 0.034 \\
-1.491 \pm 0.039\end{array}$ & $\begin{array}{r}\mathrm{I}=0.1 \mathrm{mo} \\
6.5 \pm 0.9 \\
0.0 \pm 0.7 \\
-4.8 \pm 0.8 \\
-8.7 \pm 0.9 \\
-12.3 \pm 1.0 \\
-16.3 \pm 1.0\end{array}$ & $\begin{aligned} \cdot \mathrm{kg}^{-1} & \\
1 & \pm 3 \\
-22 & \pm 2 \\
-37 & \pm 2 \\
-49 & \pm 3 \\
-59 & \pm 3 \\
-69 & \pm 3\end{aligned}$ & $\begin{array}{l}-310 \pm 18 \\
-219 \pm 6 \\
-169 \pm 5 \\
-148 \pm 5 \\
-149 \pm 4 \\
-167 \pm 5\end{array}$ \\
\hline $\begin{array}{r}0 \\
25 \\
50 \\
75 \\
100 \\
125\end{array}$ & $\begin{array}{l}-1.042 \pm 0.040 \\
-0.985 \pm 0.029 \\
-1.008 \pm 0.023 \\
-1.072 \pm 0.023 \\
-1.159 \pm 0.024 \\
-1.262 \pm 0.029\end{array}$ & $\begin{array}{r}\mathrm{I}=0.5 \mathrm{mo} \\
6.8 \pm 0.9 \\
0.6 \pm 0.8 \\
-3.8 \pm 0.8 \\
-7.2 \pm 0.9 \\
-10.2 \pm 1.0 \\
-13.4 \pm 1.1\end{array}$ & $\begin{array}{r}\cdot \mathrm{kg}^{-1} \\
5 \pm 3 \\
-17 \pm 2 \\
-31 \pm 3 \\
-41 \pm 3 \\
-50 \pm 3 \\
-58 \pm 3\end{array}$ & $\begin{array}{l}-301 \pm 18 \\
-205 \pm 6 \\
-150 \pm 5 \\
-125 \pm 5 \\
-122 \pm 5 \\
-135 \pm 5\end{array}$ \\
\hline $\begin{array}{r}0 \\
25 \\
50 \\
75 \\
100 \\
125\end{array}$ & $\begin{array}{l}-0.991 \pm 0.043 \\
-0.932 \pm 0.035 \\
-0.949 \pm 0.031 \\
-1.003 \pm 0.029 \\
-1.077 \pm 0.030 \\
-1.165 \pm 0.032\end{array}$ & $\begin{array}{r}\mathrm{I}=1.0 \mathrm{mo} \\
6.8 \pm 0.9 \\
0.9 \pm 0.9 \\
-3.2 \pm 0.8 \\
-6.1 \pm 0.8 \\
-8.7 \pm 0.8 \\
-11.3 \pm 0.8\end{array}$ & $\begin{array}{r}6 \pm 3 \\
-15 \pm 3 \\
-28 \pm 2 \\
-37 \pm 2 \\
-44 \pm 2 \\
-51 \pm 2\end{array}$ & $\begin{array}{l}-293 \pm 2 \\
-193 \pm 3 \\
-135 \pm 3 \\
-107 \pm 3 \\
-101 \pm 4 \\
-110 \pm 4\end{array}$ \\
\hline $\begin{array}{r}0 \\
25 \\
50 \\
75 \\
100\end{array}$ & $\begin{array}{l}-1.061 \pm 0.048 \\
-1.002 \pm 0.035 \\
-1.005 \pm 0.033 \\
-1.034 \pm 0.035 \\
-1.071 \pm 0.038 \\
-1.113 \pm 0.042\end{array}$ & $\begin{array}{r}\mathrm{I}=3.0 \mathrm{mo} \\
6.4 \pm 1.5 \\
1.3 \pm 1.3 \\
-1.6 \pm 1.2 \\
-3.4 \pm 1.1 \\
-4.5 \pm 1.0 \\
-5.5 \pm 1.2\end{array}$ & $\begin{array}{r}\cdot \mathrm{kg}^{-1} \\
3 \pm 5 \\
-15 \pm 4 \\
-24 \pm 4 \\
-29 \pm 3 \\
-32 \pm 3 \\
-34 \pm 3\end{array}$ & $\begin{aligned}-262 & \pm 6 \\
-154 & \pm 7 \\
-87 & \pm 8 \\
-51 & \pm 10 \\
-36 & \pm 11 \\
-36 & \pm 13\end{aligned}$ \\
\hline $\begin{array}{r}0 \\
25 \\
50 \\
75 \\
100\end{array}$ & $\begin{array}{l}-1.238 \pm 0.073 \\
-1.182 \pm 0.061 \\
-1.177 \pm 0.062 \\
-1.185 \pm 0.067 \\
-1.193 \pm 0.072 \\
-1.194 \pm 0.075\end{array}$ & $\begin{array}{r}I=5.0 \mathrm{mo} \\
5.8 \pm 2.1 \\
1.5 \pm 1.8 \\
-0.5 \pm 1.6 \\
-1.0 \pm 1.3 \\
-0.7 \pm 1.3 \\
-0.1 \pm 1.6\end{array}$ & $\begin{array}{r}\cdot \mathrm{kg}^{-1} \\
-2 \pm 7 \\
-18 \pm 6 \\
-24 \pm 5 \\
-26 \pm 5 \\
-25 \pm 4 \\
-23 \pm 5\end{array}$ & $\begin{array}{r}-234 \pm 10 \\
-119 \pm 12 \\
-45 \pm 14 \\
-1 \pm 16 \\
21 \pm 18 \\
31 \pm 21\end{array}$ \\
\hline
\end{tabular}

a Errors listed represent three times the standard deviation.

with the model presented in eq 20 . The former set comprises nine measurements made in oxalate- $\mathrm{NaCl}$ solutions with ionic strengths ranging from $0.003 \mathrm{~mol} \cdot \mathrm{kg}^{-1}$ to $0.012 \mathrm{~mol} \cdot \mathrm{kg}^{-1}$, whereas the latter set comprises eight measurements made in oxalate- $\mathrm{KCl}$ solutions with ionic strengths ranging from $0.008 \mathrm{~mol} \cdot \mathrm{kg}^{-1}$ to $0.131 \mathrm{~mol} \cdot \mathrm{kg}^{-1}$. These data were recalculated using eqs 15 and 16 after adjustment of the standard potential. McAuley and Nancollas (1961) reported the difference in potential after subtraction of the standard potential of the $\mathrm{Ag}-\mathrm{AgCl}$ electrode; the source of their standard potential values was not cited. Their potential data were used as reported. Parton and Gibbons (1939) performed their experiments in cells equipped with $\mathrm{Pt}$-quinhydrone and $\mathrm{Ag}-\mathrm{AgCl}$ electrodes; this report uses the standard potential values of Harned and Wright (1933). The resulting values of $K_{m, 1 b}$ were extrapolated independently to infinite dilution using an isothermal form of eq 20

$$
\log K_{m, 1 b}=p_{1}+p_{2} f(I)+p_{3} l(k g / m o l)-\log a_{w}
$$

The errors associated with the values of $\mathrm{pK}_{1 \mathrm{~b}}^{\ominus}$ obtained 
Table 12. Summary of Thermodynamic Quantities for the First Dissociation of Oxalic Acid in Aqueous Sodium Trifluoromethanesulfonate Media at the Saturation Vapor Pressure ${ }^{a}$

\begin{tabular}{|c|c|c|c|c|}
\hline $\mathrm{t} /{ }^{\circ} \mathrm{C}$ & $\log K_{m, 1 a}$ & $\begin{array}{c}\Delta \mathrm{H}_{1 \mathrm{a} /} \\
\left(\mathrm{kJ} \cdot \mathrm{mol}^{-1}\right)\end{array}$ & $\begin{array}{c}\Delta \mathrm{S}_{1 \mathrm{a} /} \\
\left(\mathrm{J} \cdot \mathrm{mol}^{-1} \cdot \mathrm{K}^{-1}\right)\end{array}$ & $\begin{array}{c}\Delta \mathrm{C}_{\mathrm{p}, \mathrm{la} /} \\
\left(\mathrm{J} \cdot \mathrm{mol}^{-1} \cdot \mathrm{K}^{-1}\right)\end{array}$ \\
\hline \multicolumn{5}{|c|}{$\mathrm{I}=0.1 \mathrm{~mol} \cdot \mathrm{kg}^{-1}$} \\
\hline 0 & $-1.207 \pm 0.044$ & $6.5 \pm 0.9$ & $1 \pm 3$ & $-310 \pm 18$ \\
\hline 25 & $-1.156 \pm 0.037$ & $0.0 \pm 0.7$ & $-22 \pm 2$ & $-219 \pm 6$ \\
\hline 50 & $-1.189 \pm 0.034$ & $-4.8 \pm 0.8$ & $-37 \pm 2$ & $-169 \pm 5$ \\
\hline 75 & $-1.266 \pm 0.032$ & $-8.7 \pm 0.9$ & $-49 \pm 3$ & $-148 \pm 5$ \\
\hline 100 & $-1.372 \pm 0.033$ & $-12.3 \pm 1.0$ & $-59 \pm 3$ & $-149 \pm 4$ \\
\hline 125 & $-1.496 \pm 0.038$ & $-16.3 \pm 1.0$ & $-69 \pm 3$ & $-167 \pm 5$ \\
\hline \multicolumn{5}{|c|}{$\mathrm{I}=0.5 \mathrm{~mol} \cdot \mathrm{kg}^{-1}$} \\
\hline 0 & $-1.073 \pm 0.039$ & $6.8 \pm 0.9$ & $4 \pm 3$ & $-301 \pm 18$ \\
\hline 25 & $-1.016 \pm 0.028$ & $0.6 \pm 0.8$ & $-18 \pm 2$ & $-205 \pm 6$ \\
\hline 50 & $-1.038 \pm 0.022$ & $-3.8 \pm 0.8$ & $-32 \pm 3$ & $-150 \pm 5$ \\
\hline 75 & $-1.103 \pm 0.023$ & $-7.2 \pm 0.9$ & $-42 \pm 3$ & $-125 \pm 5$ \\
\hline 100 & $-1.189 \pm 0.024$ & $-10.2 \pm 1.0$ & $-50 \pm 3$ & $-122 \pm 5$ \\
\hline 125 & $-1.292 \pm 0.029$ & $-13.4 \pm 1.1$ & $-58 \pm 3$ & $-135 \pm 5$ \\
\hline \multicolumn{5}{|c|}{$\mathrm{I}=1.0 \mathrm{~mol} \cdot \mathrm{kg}^{-1}$} \\
\hline 0 & $-1.074 \pm 0.051$ & $6.8 \pm 0.9$ & $4 \pm 3$ & $-293 \pm 2$ \\
\hline 25 & $-1.014 \pm 0.045$ & $0.9 \pm 0.9$ & $-17 \pm 3$ & $-193 \pm 3$ \\
\hline 50 & $-1.032 \pm 0.044$ & $-3.2 \pm 0.8$ & $-30 \pm 3$ & $-135 \pm 3$ \\
\hline 75 & $-1.085 \pm 0.043$ & $-6.1 \pm 0.8$ & $-38 \pm 3$ & $-107 \pm 3$ \\
\hline 100 & $-1.160 \pm 0.045$ & $-8.7 \pm 0.8$ & $-45 \pm 2$ & $-101 \pm 4$ \\
\hline 125 & $-1.247 \pm 0.047$ & $-11.3 \pm 0.8$ & $-52 \pm 3$ & $-110 \pm 4$ \\
\hline
\end{tabular}

a Errors listed represent three times the standard deviation.
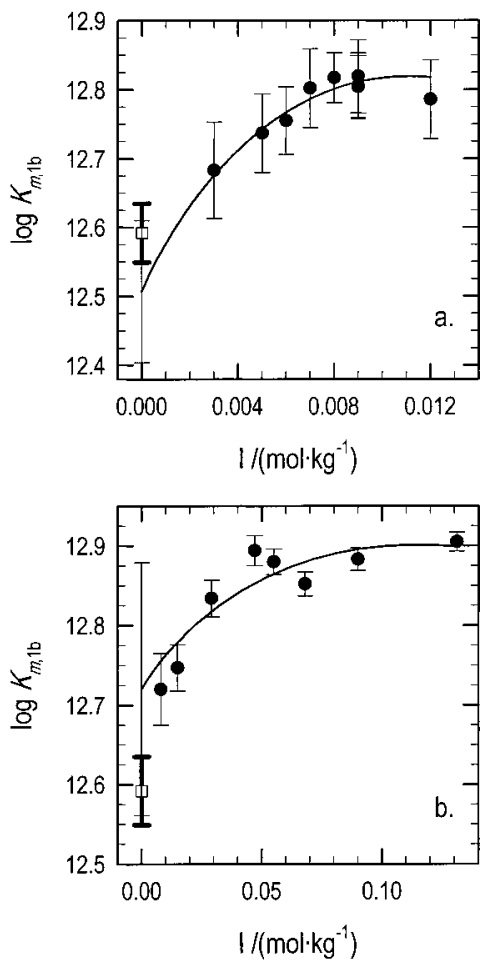

Figure 5. Plots of recalculated experimental data published by (a) McAuley and Nancollas (1961) and (b) Parton and Gibbons (1939). The experimental data are represented by the solid circles $(\bullet)$, whereas the value of $\mathrm{K}_{1 \mathrm{~b}}^{\ominus}$ obtained in this study and its associated error is designated by the open square ( $\square$ ) and bold error bar. The solid line designates the model obtained by regressing the experimental data according to eq 21 and the error in this model at I $=0 \mathrm{~mol} \cdot \mathrm{kg}^{-1}$ is shown by the thin error bar.

using these two small data sets are large: the value obtained for $\mathrm{pK}_{1 \mathrm{~b}}^{\ominus}$ using the McAuley and Nancollas data is $12.51 \pm 0.10$, whereas the results of Parton and Gibbons yield a value of $12.72 \pm 0.16$. When these errors are considered, however, the values of $\mathrm{pK}_{1 \mathrm{~b}}^{\ominus}$ obtained by these

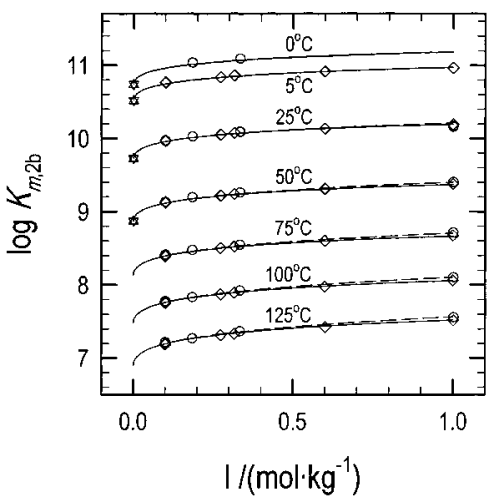

Figure 6. Relation between $\log K_{m, 2 b}$ and solution ionic strength to $1 \mathrm{~mol} \cdot \mathrm{kg}^{-1}$. The dashed curves are generated from eq 22 when solved for $\mathrm{NaCl}$ media, whereas the solid curves are generated when eq 20 is solved for $\mathrm{Na}$-triflate media. The symbols represent measurements made in $\mathrm{NaCl}$ media reported in Table $8(\bigcirc)$, measurements made in Na-triflate media reported in Table $6(\diamond)$, and the recalculated results of Harned and Fallon (1939) $(\nabla)$ and Pinching and Bates (1948) ( $\triangle$, series $1-4$ only).

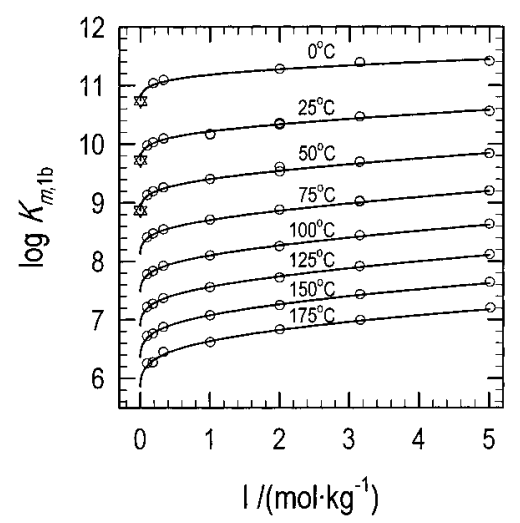

Figure 7. Relation between $\log K_{m, 2 b}$ and solution ionic strength to $5 \mathrm{~mol} \cdot \mathrm{kg}^{-1}$. The lines are generated by solving eq 22 for $\mathrm{NaCl}$ media. The symbols represent the measurements made in $\mathrm{NaCl}$ media reported in Table 8.

isothermal regressions do agree with the value of $\mathrm{pK}_{1 \mathrm{~b}}^{\ominus}$ at $25^{\circ} \mathrm{C}$ obtained in this study (12.592 \pm 0.043 ) (Figure 5). Therefore, no significant discrepancy exists between the data used to produce the generally accepted value of $\mathrm{pK}_{1 \mathrm{a}, 298}^{\ominus}$ and the model developed in this study when the former are recalculated using a more modern activity coefficient model and more recent measurements of $K_{m, 2 a}$.

Values of $\Delta \mathrm{H}_{1 b}, \Delta \mathrm{S}_{1 b}$, and $\Delta \mathrm{C}_{\mathrm{p}, 1 \mathrm{~b}}$ can be obtained by differentiation of eq 20 . These values are converted to those appropriate for the acid form of the dissociation reaction (Tables 11 and 12) by adding the corresponding values for the dissociation of water (Palmer and Drummond, 1988; Busey and Mesmer, 1978). The available enthalpy data (Table 1) are in poor agreement with those determined in this study (Christensen et al., 1967). The values of $\mathrm{pK}_{1 \mathrm{la}, 298}^{\ominus}$ used or obtained in those previous studies are significantly different from the value measured in this study. The values of $\Delta C_{p, 1 a}$ (Tables 11 and 12) obtained are identical in the two media and are equal owing to the functional form of eq 20, which invokes zero heat capacity change for the iso-Coulombic reaction.

Fitting of the Second Molal Dissociation Constant of Oxalic Acid. A model to describe the dependence of $\mathrm{K}_{\mathrm{m}, 2 \mathrm{~b}}$ as a function of temperature and ionic strength was developed by regressing the measurements made in $\mathrm{Na}$ triflate media (Table 6) simultaneously with the recalculated measurements made in $\mathrm{NaCl}$ media (Table 8) and 

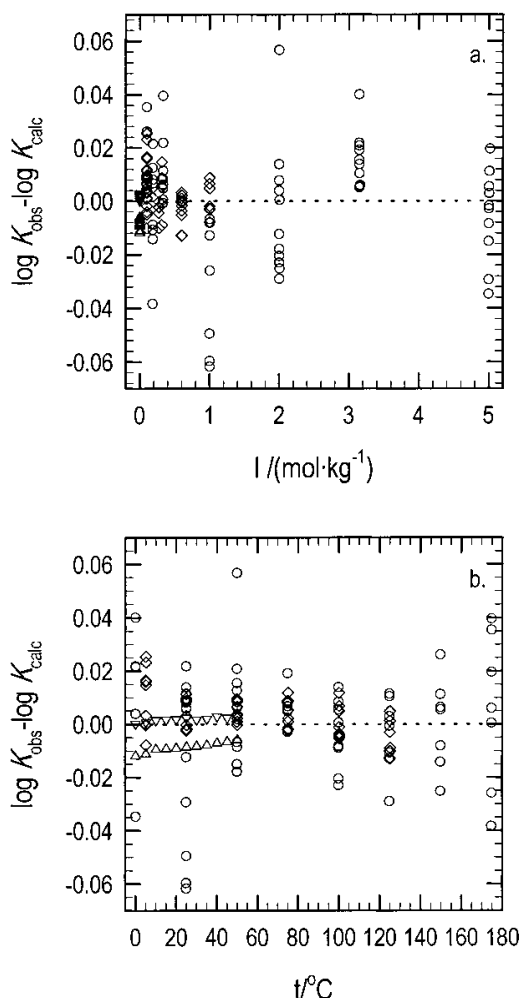

Figure 8. Deviation plots of the difference between eq 20 and the experimentally determined values of $\log _{\mathrm{m}, 2 \mathrm{~b}}$ presented in Tables 6 and 8 as a function of (a) ionic strength and (b) temperature. Symbols designate measurements made in $\mathrm{NaCl}$ media $(0$, Table 8$)$ and Na-triflate media $(\diamond$, Table 6$)$ and the recalculated measurements of Harned and Fallon (1939) $(\nabla)$ and Pinching and Bates (1948) ( $\triangle$, series 1-4 only).

values of $\mathrm{K}_{2 \mathrm{~b}}^{\ominus}$ obtained by regression of the recalculated Harned cell data (Table 10). Only a subset of these $\mathrm{K}_{2 \mathrm{~b}}^{\ominus}$ values was used; specifically, the results of the Harned and Fallon regression and the results of the Pinching and Bates series 1-4 measurements. Use of the results of all the Harned cell regressions would have given excessive weight to these previously published data. The series 1-4 measurements were used because they comprise 394 of the 617 measurements made by Pinching and Bates (1948) and were the sole data used by those authors to calculate $\mathrm{K}_{2 \mathrm{a}}^{\ominus}$.

The model obtained from this simultaneous fit is

$$
\begin{aligned}
& \mathrm{K}_{\mathrm{m}, 2 \mathrm{~b}}=2\left(\mathrm { A } _ { \phi } \left((\mathrm{I}(\mathrm{kg} / \mathrm{mol}))^{0.5} /\left(1+1.2(\mathrm{I}(\mathrm{kg} / \mathrm{mol}))^{0.5}\right)+\right.\right. \\
& \left.\left.(2 / \mathrm{l} .2) \ln \left(1+1.2(\mathrm{I}(\mathrm{kg} / \mathrm{mol}))^{0.5}\right)\right) / \mathrm{ln} 10\right)-\log \mathrm{a}_{\mathrm{w}}+\mathrm{p}_{1}+ \\
& \mathrm{p}_{2}(\mathrm{~K} / \mathrm{T})+\mathrm{p}_{3}\left(\mathrm{~T}^{2} / \mathrm{K}^{2}\right)+\mathrm{p}_{4}(\mathrm{~T} / \mathrm{K})+\mathrm{p}_{5} \mathrm{f}(\mathrm{I})+\mathrm{p}_{6} \mathrm{l}(\mathrm{kg} / \mathrm{mol})+ \\
& \mathrm{p}_{7} \mathrm{I} / \mathrm{T}(\mathrm{K} \cdot \mathrm{kg} / \mathrm{mol})+\mathrm{p}_{8} \mathrm{IT}^{2}\left(\mathrm{~kg} /\left(\mathrm{mol} \cdot \mathrm{K}^{2}\right)\right)+\mathrm{p}_{9}(\mathrm{I}(\mathrm{kg} / \mathrm{mol}))^{2}
\end{aligned}
$$

where the first term is the Debye-Hückel contribution (Pitzer, 1973), the logarithm of the standard equilibrium constant $\left(\mathrm{K}_{2 \mathrm{~b}}^{\ominus}\right)$ is defined by parameters $\mathrm{p}_{1}$ through $\mathrm{p}_{4}, \mathrm{p}_{5}$ applies to both $\mathrm{NaCl}$ and $\mathrm{Na}$-triflate media, parameters $p_{6}$ through $\mathrm{p}_{8}$ apply to $\mathrm{NaCl}$ media only, and $\mathrm{p}_{9}$ applies to $\mathrm{Na}$ triflate media only. The values for the nine parameters are $p_{1}=-7.81293, p_{2}=3946.02, p_{3}=-2.386096 \times 10^{-5}$, $\mathrm{p}_{4}=0.0215577, \mathrm{p}_{5}=-0.225671, \mathrm{p}_{6}=0.551945, \mathrm{p}_{7}=$ $-130.451, p_{8}=-1.23642 \times 10^{-6}$, and $p_{9}=-0.0159358$. Comparisons of the model shown in eq 22 and the data used in the regression are shown in Figures 6 and 7. The agreement factor for this fit is 0.80 . The residuals of the
Table 13. Summary of Thermodynamic Quantities for

\begin{tabular}{|c|c|c|c|c|}
\hline $\mathrm{t} /{ }^{\circ} \mathrm{C}$ & $\log K_{m, 2 a}$ & $\begin{array}{c}\Delta \mathrm{H}_{2 \mathrm{a}} / \\
\left(\mathrm{kJ} \cdot \mathrm{mol}^{-1}\right)\end{array}$ & $\begin{array}{c}\Delta \mathrm{S}_{2 \mathrm{a}} / \\
\left(\mathrm{J} \cdot \mathrm{mol}^{-1} \cdot \mathrm{K}^{-1}\right)\end{array}$ & $\begin{array}{c}\Delta \mathrm{C}_{\mathrm{p}, 2 \mathrm{za}} \\
\left.\mathrm{U} \cdot \mathrm{mol}^{-1} \cdot \mathrm{K}^{-1}\right)\end{array}$ \\
\hline $\begin{array}{r}0 \\
25 \\
50 \\
75 \\
100 \\
125 \\
150 \\
175\end{array}$ & $\begin{array}{l}-4.199 \pm 0.018 \\
-4.264 \pm 0.014 \\
-4.399 \pm 0.012 \\
-4.574 \pm 0.014 \\
-4.780 \pm 0.019 \\
-5.015 \pm 0.020 \\
-5.280 \pm 0.024 \\
-5.580 \pm 0.031\end{array}$ & $\begin{array}{r}\mathrm{I}=0 \mathrm{~mol} \cdot \\
-0.8 \pm 1.1 \\
-7.3 \pm 0.5 \\
-12.6 \pm 0.5 \\
-17.8 \pm 0.7 \\
-23.5 \pm 0.8 \\
-30.4 \pm 1.0 \\
-38.8 \pm 1.7 \\
-49.2 \pm 2.9\end{array}$ & $\begin{array}{r}\mathrm{kg}^{-1} \\
-83 \pm 4 \\
-106 \pm 2 \\
-126 \pm 2 \\
-143 \pm 2 \\
-160 \pm 2 \\
-180 \pm 3 \\
-202 \pm 4 \\
-162 \pm 7\end{array}$ & $\begin{array}{l}-296 \pm 44 \\
-229 \pm 27 \\
-205 \pm 18 \\
-214 \pm 13 \\
-248 \pm 19 \\
-302 \pm 32 \\
-374 \pm 54 \\
-461 \pm 73\end{array}$ \\
\hline \multicolumn{5}{|c|}{$\mathrm{I}=0.1 \mathrm{~mol} \cdot \mathrm{kg}^{-1}$} \\
\hline $\begin{array}{r}0 \\
25 \\
50 \\
75 \\
100 \\
125 \\
150 \\
175\end{array}$ & $\begin{array}{l}-3.558 \pm 0.022 \\
-3.578 \pm 0.015 \\
-3.659 \pm 0.015 \\
-3.774 \pm 0.017 \\
-3.908 \pm 0.018 \\
-4.061 \pm 0.021 \\
-4.233 \pm 0.025 \\
-4.426 \pm 0.032\end{array}$ & $\begin{array}{r}\mathrm{I}=0.5 \mathrm{mo} \\
1.6 \pm 1.2 \\
-3.9 \pm 0.6 \\
-8.0 \pm 0.5 \\
-11.6 \pm 0.7 \\
-15.4 \pm 0.8 \\
-19.8 \pm 1.0 \\
-25.2 \pm 1.6 \\
-31.8 \pm 2.8\end{array}$ & $\begin{array}{r}g^{-1} \\
-62 \pm 4 \\
-82 \pm 2 \\
-95 \pm 2 \\
-106 \pm 2 \\
-116 \pm 2 \\
-127 \pm 3 \\
-141 \pm 4 \\
-156 \pm 7\end{array}$ & $\begin{array}{l}-263 \pm 45 \\
-186 \pm 27 \\
-148 \pm 18 \\
-142 \pm 13 \\
-161 \pm 19 \\
-196 \pm 31 \\
-240 \pm 51 \\
-282 \pm 69\end{array}$ \\
\hline $\begin{array}{r}0 \\
25 \\
50 \\
75 \\
100 \\
125 \\
150 \\
175\end{array}$ & $\begin{array}{l}-3.501 \pm 0.026 \\
-3.503 \pm 0.020 \\
-3.566 \pm 0.020 \\
-3.658 \pm 0.020 \\
-3.770 \pm 0.021 \\
-3.898 \pm 0.021 \\
-4.043 \pm 0.021 \\
-4.206 \pm 0.029\end{array}$ & $\begin{array}{r}\mathrm{I}=1.0 \mathrm{mo} \\
2.6 \pm 1.3 \\
-2.7 \pm 0.7 \\
-6.4 \pm 0.6 \\
-9.6 \pm 0.6 \\
-12.8 \pm 0.6 \\
-16.6 \pm 0.7 \\
-21.3 \pm 1.5 \\
-26.8 \pm 2.7\end{array}$ & $\begin{array}{r}\cdot \mathrm{kg}^{-1} \\
-57 \pm 4 \\
-76 \pm 2 \\
-88 \pm 2 \\
-98 \pm 2 \\
-107 \pm 2 \\
-116 \pm 2 \\
-128 \pm 4 \\
-140 \pm 6\end{array}$ & $\begin{array}{l}-254 \pm 30 \\
-174 \pm 24 \\
-133 \pm 17 \\
-124 \pm 12 \\
-138 \pm 18 \\
-168 \pm 30 \\
-205 \pm 45 \\
-236 \pm 61\end{array}$ \\
\hline $\begin{array}{r}0 \\
25 \\
50 \\
75 \\
100 \\
125 \\
150 \\
175\end{array}$ & $\begin{array}{l}-3.591 \pm 0.053 \\
-3.540 \pm 0.031 \\
-3.548 \pm 0.032 \\
-3.587 \pm 0.035 \\
-3.640 \pm 0.038 \\
-3.707 \pm 0.039 \\
-3.787 \pm 0.043 \\
-3.880 \pm 0.057\end{array}$ & $\begin{array}{r}\mathrm{I}=3.0 \mathrm{mo} \\
5.7 \pm 2.6 \\
1.0 \pm 2.0 \\
-2.1 \pm 1.4 \\
-4.4 \pm 1.2 \\
-6.5 \pm 1.3 \\
-9.1 \pm 1.9 \\
-12.2 \pm 2.9 \\
-15.7 \pm 4.5\end{array}$ & $\begin{array}{r}\cdot \mathrm{kg}^{-1} \\
-48 \pm 9 \\
-65 \pm 6 \\
-74 \pm 5 \\
-81 \pm 4 \\
-87 \pm 4 \\
-94 \pm 5 \\
-101 \pm 7 \\
-1091 \pm 1\end{array}$ & $\begin{array}{r}-233 \pm 38 \\
-150 \pm 35 \\
-102 \pm 32 \\
-85 \pm 32 \\
-92 \pm 37 \\
-112 \pm 47 \\
-134 \pm 61 \\
-146 \pm 78\end{array}$ \\
\hline $\begin{array}{r}0 \\
25 \\
50 \\
75 \\
100 \\
125 \\
150 \\
175\end{array}$ & $\begin{array}{l}-3.809 \pm 0.095 \\
-3.711 \pm 0.062 \\
-3.676 \pm 0.060 \\
-3.670 \pm 0.067 \\
-3.680 \pm 0.070 \\
-3.701 \pm 0.071 \\
-3.734 \pm 0.078 \\
-3.778 \pm 0.100\end{array}$ & $\begin{array}{r}I=5.0 \mathrm{mo} \\
8.3 \pm 4.2 \\
4.0 \pm 3.2 \\
1.3 \pm 2.4 \\
-0.4 \pm 1.8 \\
-1.9 \pm 2.1 \\
-3.7 \pm 3.2 \\
-5.9 \pm 4.9 \\
-8.2 \pm 7.0\end{array}$ & $\begin{aligned} \cdot \mathrm{kg}^{-1} & \\
-421 & \pm 4 \\
-581 & \pm 1 \\
-66 & \pm 8 \\
-71 & \pm 6 \\
-76 & \pm 6 \\
-80 & \pm 8 \\
-851 & \pm 2 \\
-911 & \pm 7\end{aligned}$ & $\begin{array}{r}-221 \pm 48 \\
-135 \pm 48 \\
-82 \pm 50 \\
-61 \pm 54 \\
-63 \pm 61 \\
-77 \pm 73 \\
-92 \pm 86 \\
-93 \pm 103\end{array}$ \\
\hline
\end{tabular}
the Second Dissociation of Oxalic Acid in Aqueous Sodium Chloride Media at the Saturation Vapor Pressure $^{a}$

a Errors listed represent three times the standard deviation.

regression exhibit no systematic relationship to ionic strength or temperature (Figure 8). Values of $\mathrm{K}_{\mathrm{m}, 2 \mathrm{a}}$ are derived from eq 22 and the appropriate values for the dissociation of water (Palmer and Drummond, 1988; Busey and Mesmer, 1978). These values are reported in Tables 13 and 14 and are in good agreement with the recalculated Harned cell data, particularly with the recalculated Harned and Fallon (1939) measurements. Whereas eq 22 is strictly valid for Na-triflate solutions at temperatures up to 125 ${ }^{\circ} \mathrm{C}$ and values of ionic strength to $1.0 \mathrm{~mol} \cdot \mathrm{kg}^{-1}$, the limits in $\mathrm{NaCl}$ solutions are $175^{\circ} \mathrm{C}$ and $5.0 \mathrm{~mol} \cdot \mathrm{kg}^{-1}$, respectively. 


\section{Table 14. Summary of Thermodynamic Properties for the Second Dissociation of Oxalic Acid in Aqueous Sodium Trifluoromethanesulfonate Media at the Saturation Vapor Pressure of Water ${ }^{\mathrm{a}}$}

\begin{tabular}{|c|c|c|c|c|}
\hline${ }^{\circ} \mathrm{C}$ & $\log K_{m, 2 a}$ & $\begin{array}{c}\Delta \mathrm{H}_{2 \mathrm{a}} / \\
\left(\mathrm{kJ} \cdot \mathrm{mol}^{-1}\right)\end{array}$ & $\begin{array}{c}\Delta \mathrm{S}_{2 \mathrm{a}} / \\
\left(\mathrm{J} \cdot \mathrm{mol}^{-1} \cdot \mathrm{K}^{-1}\right)\end{array}$ & $\begin{array}{c}\Delta \mathrm{C}_{\mathrm{p}, 2 \mathrm{a}} / \\
\left.\mathrm{J} \cdot \mathrm{mol}^{-1} \cdot \mathrm{K}^{-1}\right)\end{array}$ \\
\hline $\begin{array}{r}0 \\
25 \\
50 \\
75 \\
100 \\
125\end{array}$ & $\begin{array}{l}-3.768 \pm 0.010 \\
-3.814 \pm 0.005 \\
-3.924 \pm 0.006 \\
-4.072 \pm 0.006 \\
-4.247 \pm 0.008 \\
-4.441 \pm 0.009\end{array}$ & $\begin{array}{r}\mathrm{I}=0.1 \mathrm{mo} \\
-0.5 \pm 0.9 \\
-6.5 \pm 0.4 \\
-11.7 \pm 0.3 \\
-16.6 \pm 0.4 \\
-21.8 \pm 0.4 \\
-27.7 \pm 0.6\end{array}$ & $\begin{aligned} \cdot \mathrm{kg}^{-1} & \\
-74 & \pm 3 \\
-95 & \pm 1 \\
-111 & \pm 1 \\
-126 & \pm 1 \\
-140 & \pm 1 \\
-154 & \pm 2\end{aligned}$ & $\begin{array}{l}-259 \pm 27 \\
-221 \pm 20 \\
-200 \pm 13 \\
-199 \pm 8 \\
-218 \pm 14 \\
-257 \pm 27\end{array}$ \\
\hline $\begin{array}{r}0 \\
25 \\
50 \\
75 \\
100 \\
125\end{array}$ & $\begin{array}{l}-3.532 \pm 0.012 \\
-3.564 \pm 0.009 \\
-3.655 \pm 0.009 \\
-3.779 \pm 0.008 \\
-3.924 \pm 0.008 \\
-4.084 \pm 0.009\end{array}$ & $\begin{array}{r}\mathrm{I}=0.5 \mathrm{mo} \\
-0.3 \pm 0.9 \\
-6.0 \pm 0.4 \\
-11.0 \pm 0.3 \\
-15.5 \pm 0.4 \\
-20.1 \pm 0.4 \\
-25.4 \pm 0.6\end{array}$ & $\begin{array}{r}-88 \pm 1 \\
-104 \pm 1 \\
-117 \pm 1 \\
-129 \pm 1 \\
-142 \pm 2\end{array}$ & $\begin{array}{l}-248 \pm 27 \\
-210 \pm 20 \\
-186 \pm 13 \\
-182 \pm 8 \\
-197 \pm 14 \\
-230 \pm 27\end{array}$ \\
\hline $\begin{array}{r}0 \\
25 \\
50 \\
75 \\
100 \\
125\end{array}$ & $\begin{array}{l}-3.486 \pm 0.021 \\
-3.513 \pm 0.019 \\
-3.594 \pm 0.019 \\
-3.705 \pm 0.019 \\
-3.834 \pm 0.019 \\
-3.974 \pm 0.020\end{array}$ & $\begin{array}{r}\mathrm{I}=1.0 \mathrm{mo} \\
-0.3 \pm 0.9 \\
-5.8 \pm 0.4 \\
-10.5 \pm 0.3 \\
-14.8 \pm 0.4 \\
-19.2 \pm 0.4 \\
-24.1 \pm 0.6\end{array}$ & $\begin{aligned} \mathrm{kg}^{-1} & \\
-68 & \pm 3 \\
-87 & \pm 1 \\
-101 & \pm 1 \\
-113 & \pm 1 \\
-125 & \pm 1 \\
-137 & \pm 2\end{aligned}$ & $\begin{array}{l}-241 \pm 27 \\
-203 \pm 20 \\
-177 \pm 13 \\
-171 \pm 8 \\
-183 \pm 14 \\
-213 \pm 27\end{array}$ \\
\hline
\end{tabular}

a Errors listed represent three times the standard deviation.

Numerical differentiation of eq 22 using the ORGLS program yields $\Delta \mathrm{H}_{2 b}, \Delta \mathrm{S}_{2 \mathrm{~b}}$, and $\Delta \mathrm{C}_{\mathrm{p}, 2 \mathrm{~b}}$ which were converted to the equivalent values for the acid form (Tables 13 and 14). Although the value obtained for $\Delta \mathrm{H}_{2 a}^{\circ}$ at 25 ${ }^{\circ} \mathrm{C}$ is not in good agreement with the value reported by Christensen et al. (1967), it is preferred because it is constrained tightly by the precise Harned cell measure ments (Table 10). On the other hand, the titration calorimetry experiments were performed over a very narrow temperature range, and $\Delta \mathrm{H}$ was assumed to be independent of I over the narrow range of ionic strength in which the titrations were performed (Christensen et al., 1967).

\section{Conclusions}

Although the revisions proposed here to the generally accepted values describing the dissociation of oxalic acid are relatively minor, they do clarify the apparently anomalous behavior of the dissociation constants of oxalic acid at low temperatures. Previous work in this laboratory (Mesmer et al., 1989; Bell et al., 1993; Kettler et al., 1991, 1992, 1995a,b) has shown that most carboxylic acids become increasingly dissociated with increasing temperature followed by reassociation at higher temperatures (viz., the temperature dependence of $\log K_{m, n a}$ reverses with increasing temperature). As the concentration of a supporting electrolyte increases, the temperature at which this reversal occurs also increases. The reassociation is driven by the decline in the value of $T \Delta S_{a}$ that is a consequence of hydration of the product ions (Mesmer et al., 1988). The negative value of $T \Delta S_{a}$ is offset by $\Delta \mathrm{H}_{a}$ in solutions with high ionic strength and low temperatures: $\Delta H_{a}$ is dominated by the breaking of the $\mathrm{H}$-anion bond rather than by the hydration of the product ions (Mesmer et al., 1988). The first dissociation of oxalic acid had been an exception to this trend: values of $\log K_{m, 1 a}$ became more negative with increasing temperature irrespective of the solution ionic strength (Kettler et al., 1991; McAuley and Nancollas, 1961; Kurz and Farrar, 1969). The data in Tables 11 and 12 are evidence that the behavior of oxalic acid is not anomalous, but rather that previously published schemes were inadequate for treating a diprotic acid with closely spaced $\mathrm{pK}_{1 \mathrm{a}}^{\ominus}$ and $\mathrm{pK}_{1 \mathrm{~b}}^{\ominus}$ values; a problem exacerbated by the relatively low $\mathrm{pK}_{1 \mathrm{a}}^{\ominus}$ value.

The differences in the values of log $K_{m, n a}$ in the two different ionic media are very small and exceed the assigned errors only at temperatures above $50{ }^{\circ} \mathrm{C}$ and values of ionic strength of $1 \mathrm{~mol} \cdot \mathrm{kg}^{-1}$. The small differences result from similarities in $\Delta \mathrm{S}_{1 \mathrm{a}}$. On the other hand, values of $\Delta \mathrm{H}_{2 a}$ and $\Delta \mathrm{S}_{2 a}$ in the two media do differ by amounts exceeding the combined errors even when the values of $\log \mathrm{K}_{\mathrm{m}, 2 a}$ are not significantly different. Values of $\Delta \mathrm{H}_{2 a}$ obtained in $\mathrm{NaCl}$ media are greater than those obtained in Na-triflate media. The difference in $\Delta \mathrm{H}_{2 a}$ values is partially offset by the differences in $\Delta \mathrm{S}_{2 a}$ values; the latter are also greater in $\mathrm{NaCl}$ media than in $\mathrm{Na}$-triflate media.

The results of this study revise the generally accepted values for the dissociation of oxalic acid at low temperatures and ionic strengths and present some new measurements for the dissociation of oxalic acid in Na-triflate media. These revisions clarify the thermodynamics of the first dissociation of oxalic acid and are evidence that oxalic acid behaves similarly to other carboxylic acids in aqueous solutions. The measurement of the molal dissociation constants of oxalic acid in $\mathrm{Na}$-triflate solutions provides the information necessary to measure the association quotients of a variety of metal-oxalate complexes in a noncomplexing electrolyte.

\section{Literature Cited}

Ayres, J. A. Decontamination of nuclear reactors and equipment Ronald Press: New York, 1970

Baes, C. F., J r.; Mesmer, R. E. The Hydrolysis of Cations; Wiley: New York, 1976.

Bates, R. G. Determination of pH, Theory and Practice; Wiley: New York, 1964.

Bauer, R. F.; Smith, W. M. The mono-oxalato complexes of iron(III). Part I. Equilibria. Can. J . Chem. 1965, 43, 2755-2762.

Bayri, B.; Rosset, R.; Desbarres, J .; J ardy, A.; Noel, D.; Kerrec, O. Lantes, B. Complexing properties of the main organic acids used in decontamination solutions for nuclear power plants and reactions involving their degradation or elimination. Nucl. Eng. Des. 1996, 160, 159-170.

Bell, J . L. S.; Wesolowski, D. J .; Palmer, D. A. The dissociation of formic acid in sodium chloride solutions to $200^{\circ} \mathrm{C}$. J . Solution Chem. 1993, 22, 125-136.

Bénézeth, P.; Palmer, D. A.; Wesolowski, D. J . Dissociation quotients for citric acid in aqueous sodium chloride media to $150{ }^{\circ} \mathrm{C}$. J . Solution Chem. 1997, 26, 63-84.

Bottari, E.; Ciavatta, L. Potentiometric study of iron(II)-oxalate complexes. Gazz. Chim. Ital. 1965, 95, 908-920.

Busey, R. H.; Mesmer, R. E. The ionization of water in $\mathrm{NaCl}$ media to $300{ }^{\circ} \mathrm{C}$. J . Solution Chem. 1976, 5, 147-152.

Busey, R. H.; Mesmer, R. E. Thermodynamic quantities for the ionization of water in $\mathrm{NaCl}$ media to $300^{\circ} \mathrm{C}$. J . Chem. Eng. Data 1978, 23, 175-176.

Busing, W. R.; Levy, H. A. ORGLS: A general fortran least-square program; Oak Ridge National Laboratory Report, ORNL-TM (U.S.) ORNL-TM-271, Oak Ridge National Laboratory, Oak Ridge, TN, 1963.

Choppin, G. R.; Chen, J. F. Complexation of Am(III) by oxalate in $\mathrm{NaClO}_{4}$ media. Radiochim. Acta 1996, 74, 105-110.

Christensen, J. J .; I zatt, R. M.; Hansen, L. D. Thermodynamics of proton ionization in dilute aqueous solution. VII. $\Delta \mathrm{H}^{\circ}$ and $\Delta \mathrm{S}^{\circ}$ values for proton ionization from carboxylic acids at $25^{\circ}$. J . Am Chem. Soc. 1967, 89, 213-222.

Cruywagen, J .J .; Heyns, J . B.; van der Water, R. F. A potentiometric, spectrophotometric, and calorimetric investigation of molybdenum(VI)-oxalate complex formation. J . Chem. Soc., Dalton Trans. 1986, 1857-1862.

Darken, L. S. The ionization constants of oxalic acid at $25{ }^{\circ} \mathrm{C}$ from conductance measurements. J. Am. Chem. Soc. 1941, 63, 10071011.

de Bruin, H. J .; Kairaitis, D.; Temple, R. B. The extraction of anionic beryllium complexes by tri-iso-octylamine. Aust. J . Chem. 1962, 15, 457-466.

Dickson, A.; Wesolowski, D. J .; Palmer, D. A.; Mesmer, R. E. Dissociation constant of bisulfate in aqueous sodium chloride solutions to $250{ }^{\circ} \mathrm{C}$. J . Phys. Chem. 1990, 94, 7978-7985. 
Graustein, W. C.; Cromack, K., J r.; Sollins, P. Calcium oxalate: Occurrence in soils and effect on nutrient and geochemical cycles. Science 1977, 198, 1252-1254.

Harned, H. S.; Wright, D. D. A study of the cell, Pt/quinhydrone, $\mathrm{HCl}$ $(0.01 \mathrm{M}) / \mathrm{AgCl} / \mathrm{Ag}$, and the normal electrode potential of the quinhydrone electrode from 0 to $40^{\circ}$. J . Am. Chem. Soc. 1933, 55, 48494857.

Harned, H. S.; Fallon, L. D. The second ionization constant of oxalic acid from 0 to $50^{\circ} \mathrm{C}$. J . Am. Chem. Soc. 1939, 61, 3111-3113.

Ho, P. C.; Palmer, D. A. Electrical conductances of dilute aqueous trifluoromethanesulfonate solutions at temperatures $0-450^{\circ} \mathrm{C}$ and pressure up to $250 \mathrm{MPa}$. J. Solution Chem. 1995, 24, 753-769.

Kettler, R. M.; Palmer, D. A.; Wesolowski, D. J . Dissociation quotients of oxalic acid in aqueous sodium chloride media to $175{ }^{\circ} \mathrm{C}$. J Solution Chem. 1991, 20, 905-927.

Kettler, R. M.; Wesolowski, D. J .; Palmer, D. A. Dissociation quotients of malonic acid in aqueous sodium chloride media to $100{ }^{\circ} \mathrm{C}$. J . Solution Chem. 1992, 21, 883-900.

Kettler, R. M.; Wesolowski, D. J .; Palmer, D. A. Dissociation quotient of benzoic acid in aqueous sodium chloride media to $250{ }^{\circ} \mathrm{C}$. J . Solution Chem. 1995a, 24, 385-407.

Kettler, R. M.; Palmer, D. A.; Wesolowski, D. J . Dissociation quotients of succinic acid in aqueous sodium chloride media to $250^{\circ} \mathrm{C}$. J . Solution Chem. 1995b, 24, 65-87.

Kurz, J. L.; Farrar, J. M. The entropies of dissociation of some moderately strong acids. J. Am. Chem. Soc. 1969, 91, 6057-6062.

Lindsay, W. T., J r. Estimation of concentration quotients for ionic equilibria in high temperature water. Proc. Int. Water Conf. Eng. Soc. W. Pa. 1980, 41, 284-294.

Maeda, M.; Hisada, O.; Kinjo, Y.; Ito, K. Estimation of salt and temperature effects on ion product of water in aqueous solution. Bull. Chem. Soc. J pn. 1987, 60, 3233-3239.

Martell, A. E.; Smith, R. M. Critical Stability Constants. Volume 3: Other Organic Ligands; Plenum: New York, 1977.

McAuley, A.; Nancollas, G. H. Complex formation in solutions of copper oxalate. Trans. Faraday Soc. 1960, 56, 1165-1171.

McAuley, A.; Nancollas, G. H. Thermodynamics of ion association. Part VII. Some transition- metal oxalates. J . Chem. Soc. A 1961, 22152221.

Mesmer, R. E.; Holmes, H. F. pH, definition and measurement at high temperatures. J. Solution Chem. 1992, 21, 725-744.

Mesmer, R. E.; Baes, C. F., J r.; Sweeton, F. H. Acidity measurements at elevated temperatures. IV. Apparent dissociation of water in 1 m potassium chloride up to $292^{\circ} \mathrm{C}$. J . Phys. Chem. 1970, 74, 19371942.

Mesmer, R. E.; Marshall, W. L.; Palmer, D. A.; Simonson, J. M.; $\mathrm{Holmes}, \mathrm{H}$. F. Thermodynamics of aqueous association and ionization reactions at high temperatures and pressures. J. Solution Chem. 1988, 17, 699-718.

Mesmer, R. E.; Patterson, C. S.; Busey, R. H.; Holmes, H. F. I onization of acetic acid in $\mathrm{NaCl}$ (aq) media: A potentiometric study to $573 \mathrm{~K}$ and 130 bar. J. Phys. Chem. 1989, 93, 7483-7490.

Mills, I.; Cvitaš, T.; Homann, K.; Kallay, N.; Kuchitsu, K. Quantities, units and symbols in physical chemistry; Blackwell Scientific Publications: Oxford, 1993.
Moorhead, E. G.; Sutin, N. Rate and equilibrium constants for the formation of the monooxalate complex of iron (III). Inorg. Chem. 1966, 5, 1866-1871.

Palmer, D. A.; Drummond, S. E. The molal dissociation quotients of water in sodium trifluoromethanesulfonate solutions to high temperatures. J. Solution Chem. 1988, 17, 153-164.

Palmer, D. A.; Hyde, K. E. Ferrous chloride and acetate complexation in aqueous solutions at high temperatures. Geochim. Cosmochim. Acta 1993, 57, 1393-1408.

Palmer, D. A.; Wesolowski, D. J . Aluminum speciation and equilibria in aqueous solution. III. Potentiometric determination of the first hydrolysis constant of aluminum(III) in sodium chloride solutions to $125^{\circ} \mathrm{C}$. Geochim. Cosmochim. Acta 1993, 57, 2929-2938.

Parton, H. N.; Gibbons, R. C. The thermodynamic dissociation constants of oxalic acid. Trans. Faraday Soc. 1939, 35, 542-545.

Pinching, G. D.; Bates, R. G. Second dissociation constant of oxalic acid from $0^{\circ}$ to $50^{\circ} \mathrm{C}$, and the $\mathrm{pH}$ of certain oxalate buffer solutions. J. Res. Natl. Bur. Stand. (U.S.) 1948, 40, 405-416.

Pitzer, K. S. Thermodynamics of electrolytes. I. Theoretical basis and general equations. J. Phys. Chem. 1973, 77, 268-277.

Quist, A. S.; Marshall, W. L. Assignment of limiting equivalent conductances for single ions to $400^{\circ} \mathrm{C}$. J . Phys. Chem. 1965, 69, 2984-2987.

Rajan, K. S.; Martell, A. E. Equilibrium studies of uranyl complexesIV. Reactions with carboxylic acids. J . Inorg. Nucl. Chem. 1967, 29, 523-529.

Robinson, R. A.; Stokes, R. H. Electrolyte solutions; Butterworth: London, 1959 .

Ubaldini, S.; Piga, L.; Fornari, P.; Massidda, R. Removal of iron from quartz sands: A study of column leaching using a complete factorial design. Hydrometallurgy 1996, 40, 369-379.

Vegliò, F.; Passariello, B.; Toro, L.; Marabini, A. M. Development of a bleaching process for a kaolin of industrial interest by oxalic ascorbic, and sulfuric acids: Preliminary study using statistical methods of experimental design. Ind. Eng. Chem. Res. 1996, 35 1680-1687.

Wesolowski, D. J .; Drummond, S. E.; Mesmer, R. E.; Ohmoto, H. Hydrolysis equilibria of tungsten(VI) in aqueous sodium chloride solutions to $300^{\circ} \mathrm{C}$. I norg. Chem. 1984, 23, 1120-1132.

Received for review August 12, 1997. Accepted J anuary 22, 1998. This study was performed while R.M.K. was a summer faculty participant with the Oak Ridge Institute for Science and Education. Financial support for this project was provided by the Office of Basic Energy Sciences of the U.S. Department of Energy under Contract DE-AC05-960R22464 with Lockheed Martin Energy Research Corporation. R.M.K. received additional support from the National Science Foundation (EAR 9317075) and the Research Council of the University of Nebraska.

\section{JE970195Z}

